\title{
Formation and Dynamics of Transequatorial Loops
}

\author{
Avyarthana Ghosh and Durgesh Tripathi
}

Inter-University Centre for Astronomy and Astrophysics, Post Bag - 4, Ganeshkhind, Pune 411007, India

e-mail: avyarthana@iucaa.in ; durgesh@iucaa.in

May 27, 2020

\section{ABSTRACT}

\begin{abstract}
Aims. To study the dynamical evolution of trans-equatorial loops (TELs) using imaging and spectroscopy.
Methods. We have used the images recorded by the Atmospheric Imaging Assembly and the Helioseismic Magnetic Imager on-board the Solar Dynamics Observatory and spectroscopic observations taken from the Extreme-Ultraviolet Imaging Spectrometer on-board Hinode.

Results. The data from AIA $193 \AA$ A channel show that TELs are formed between AR 12230 and a newly emerging AR 12234 and evolved during December 10-14, 2014. The xt-plots for December 12, 2014 obtained using AIA $193 \AA$ A data reveal signatures of inflow and outflow towards an X-region. High cadence AIA images also show recurrent intensity enhancements in close proximity to the X-region (P2), which is observed to have higher intensities for spectral lines formed at $\log T[K]=6.20$ and voids at other higher temperatures. The electron densities and temperatures in the X-region (and P2) are maintained steadily at $\log N_{e}=8.5-8.7 \mathrm{~cm}^{-3}$ and $\log T[K]=6.20$, respectively. Doppler velocities in the X-region show predominant redshifts by about $5-8 \mathrm{~km} \mathrm{~s}^{-1}$ when closer to the disk centre but blueshifts (along with some zero-velocity pixels) when away from the centre. The Full-Width-Half-Maxima (FWHM) maps reveal non-thermal velocities of about $27-30 \mathrm{~km} \mathrm{~s}^{-1}$ for Fe xII, Fe xIII and Fe xv lines. However, the brightest pixels have nonthermal velocities $\sim 62 \mathrm{~km} \mathrm{~s}^{-1}$ for Fe xII and Fe xIII lines. On the contrary, the dark X-region for Fe xv line have the highest non-thermal velocity $\left(\sim 115 \mathrm{~km} \mathrm{~s}^{-1}\right)$.

Conclusions. We conclude that the TELs are formed due to magnetic reconnection. We further note that the TELs themselves undergo magnetic reconnection leading to reformation of loops of individual ARs. Moreover, this study, for the first time, provides measurements of plasma parameters in X-regions thereby providing essential constraints for theoretical studies.
\end{abstract}

Key words. Sun - activity - photosphere - corona - magnetic fields - sunspots - flares

\section{Introduction}

It is well established that the solar corona is full of loop structures (see, e.g., Reale 2014 , for a review) as well as diffuse emissions (Del Zanna \& Mason 2003; Tripathi et al. 2009, Viall \& Klimchuk 2011; Subramanian et al. 2014). One of those are the trans-equatorial loops (TELs), which join ARs across the equator of the Sun. The theoretical existence of such loop structures was first suggested by Babcock (1961) as a consequence of the solar dynamo. However, such loops were first reported over a decade later in 1974 using the observations recorded by the X-Ray Telescope (XRT) on-board Skylab (Vaiana et al.1974). Based on the subsequent observations of ARs associated with McMath plage numbers 12472 and 12474, Chase et al. (1976) and Svestka et al. (1977) suggested that the TELs were formed due to magnetic reconnection between two ARs.

Using the observations recorded by the Soft X-ray Telescope (Tsuneta et al. 1991) on-board Yohkoh, Tsuneta (1996) reported the formation of X-type topology in TELs connecting two ARs in two hemispheres. This has been followed by a number of authors (Fárník et al. 1999: Pevtsov 2000: Fárník et al. 2001:Fárník \& Švestka 2002; Crooker et al. 2002; Pevtsov 2004; Chen et al. 2006, 2007; Wang et al. 2007; Shimojo et al. 2007; Yokoyama \& Masuda 2009, 2010) reporting the formation, characteristics and evolution of TELs, using the observations taken from SXT as well as the X-Ray Telescope (XRT; Golub et al. 2007) on-board Hinode (Kosugi et al. 2007).
The early spectroscopic diagnostics of TELs were reported by authors viz., Harra et al. (2003) and Brosius (2006) using the observations recorded by the Coronal Diagnostic Spectrometer (CDS; Harrison et al. 1995) on-board the Solar and Heliospheric Observatory (SOHO; Domingo et al. 1995). However, we emphasize that these spectroscopic measurements were performed in and along the off-limb TELs only.

$\mathrm{X}$-shaped regions (cusp regions) are believed to be signatures of magnetic reconnection, for e.g., Tsuneta et al. (1992); Forbes \& Acton (1996); Tripathi et al. (2006, 2007). Therefore, measurement of plasma parameters in the $\mathrm{X}$-shaped structure formed between the TELs may help us probe the physical properties of reconnection regions. To the best of our knowledge, there are no such measurements till date for TELs.

In this paper, we perform a detailed study of a complete sequence of the formation and evolution of a set of TELs using the observations taken from the Atmospheric Imaging Assembly (AIA; Lemen et al. 2012) and the Helioseismic Magnetic Imager (HMI; Schou et al. 2012a b) on-board the Solar Dynamics Observatory (SDO; Boerner et al. 2012; Pesnell et al.2012). This is accompanied with spectroscopic observations recorded with the Extreme-ultraviolet Imaging Spectrometer (EIS; Culhane et al. 2007) on-board Hinode. The rest of the paper is structured as follows. In $\$ 2$, we discuss the observations used for this study. Data analysis and results are presented in $\$ 3$ Finally, we summarize and discuss the results in $\$ 4$. 


\section{Observations and data}

In this work, we have primarily used the AIA and EIS observations. AIA provides near-simultaneous full-disk observations of the solar atmosphere in 7 EUV channels sensitive to different temperatures (O'Dwyer et al. 2010, Del Zanna et al. 2011; Boerner et al. 2012) with an approximate time cadence of $12 \mathrm{~s}$ and a pixel size of $0.6^{\prime \prime}$. EIS provides spectroscopic observations in two wavelength bands, CCD A (170-210 ̊) and CCD B (250-290 $\AA$ ) using four slit widths $1,2,4$ and $250^{\prime \prime}$. We have used the AIA observations to study the long term evolution of the TELs and EIS observations to measure the physical plasma parameters such as electron density, temperature, Doppler velocities and non-thermal widths.

A portion of the solar disk observed on the AIA $193 \AA$ channel on December 12, 2014 at 19:35:30 UT, is shown in panel A of Fig. 1. The over-plotted green box indicates the regionof-interest (ROI) and displayed in panel B and considered for further study. The over-plotted white box in both panels A \& $\mathrm{B}$ shows the EIS raster field-of-view (FOV). In panel B, we overplot two white dashed lines crossing each other to highlight the X-shaped structure. Fortuitously, the top part of the EIS raster FOV covers this $\mathrm{X}$-shaped region. Also, the blue (positive) and black (negative) contours represent the Line-Of-Sight (LOS) magnetic flux density of $500 \mathrm{G}$ as observed at a nearsimultaneous time (19:35:40 UT) by HMI. The same HMI image is shown in panel (C) where we clearly see two bipolar active regions ( $A R 12230$ and $A R$ 12234) on either side of the solar equator.

We note that EIS rastered this region four times during 10:16:00-11:17:00 UT, 19:04:33-20:05:25 UT, 20:40:0021:41:30 UT and 22:16:00-23:17:00 UT on December 12th, 2014 using the $2^{\prime \prime}$ slit. We denote these rasters as 'E1', 'E2', 'E3' and 'E4', respectively, where E2 is the closest to the disk center. All these rasters are 60-steps rasters that covered a FOV of $120^{\prime \prime} \times 512^{\prime \prime}$. We have processed all the AIA and EIS observations with the standard software provided in Solarsoft (Freeland \& Handy 1998).

Table 1. EIS spectral lines used for studying the plasma parameters at the reconnection region, as well as the adjacent loops where $\lambda_{0}$ is the rest wavelength. The peak formation temperatures are taken from CHIANTI (Dere et al. 1996), Del Zanna et al.2015) at one particular density.

\begin{tabular}{|c|c|c|}
\hline Ion name & $\lambda_{0}(\AA)$ & $\log \mathrm{T}_{\text {peak }}$ \\
& (Brown et al. 2008$)$ & {$[\mathrm{K}]$} \\
\hline Fe VIII & $185.213^{t, w}$ & 5.80 \\
Si VII & $275.368^{w, * *}$ & 5.80 \\
Fe IX & $197.862^{t}$ & 5.90 \\
Fe X & $184.536^{t}$ & 6.05 \\
Fe XI & $180.401^{a, t}, 182.167^{b}$ & 6.10 \\
Fe XII & $186.880^{a}, 192.394^{t}, 195.119^{b, t}$ & 6.20 \\
Fe XIII & $202.044^{a, t}, 203.826^{b}$ & 6.25 \\
Fe XIV & $264.787^{a, t}, 270.519^{b}$ & 6.30 \\
Fe XV & $284.160^{t}$ & 6.35 \\
\hline
\end{tabular}

\footnotetext{
${ }^{a, b}$ density sensitive line pair

${ }^{t}$ used for temperature diagnostics

${ }^{w}$ used for deriving the reference wavelength

** Taken from Warren et al. (2011)
}

The EIS rasters included several spectral lines spread over a broad range of temperatures $(\log [T / K]=5.80-6.35)$. Table 1 lists the spectral lines along with their laboratory wavelengths taken from Brown et al. (2008) and peak formation temperatures derived from CHIANTI (Dere et al. 1996, Del Zanna et al.2015). The reference wavelength for Doppler velocities are derived using the relatively cooler lines, Fe vIII and Si vII for CCD A and CCD B, respectively. For this purpose, we have used the averaged spectra for all pixels belonging to rows 50-149 (from the bottom) of the EIS raster (highlighted by the white box in panels A and B of Fig. 1) because these represent the quiet Sun better. However, we have ignored the bottom 0-49 rows due to missing data. We have used the eis_auto_fit.pro package $e^{1}$ that rectifies the EIS spectral data by removing the errors due to orbital drift and slit tilt. An uncertainty of $\sim 5 \mathrm{~km} \mathrm{~s}^{-1}$ is expected on EIS velocity estimates 2

We note that there are four line pairs, viz., Fe XI, Fe XII, Fe XIII and Fexiv (labelled with a, b in Table 1), which are suitable to estimate the electron densities at four different temperatures (see e.g., Young et al. 2007, Tripathi et al. 2010, and reference therein). For Fe XII, the spectral line identified at $186.88 \AA$ has two components at $186.854 \AA$ and $186.887 \AA$. Moreover, Fe XII $195.119 \AA$ line has a self-blend at $195.179 \AA$, which is significant (> 5\%) only at $\log N_{e}>9.5 \mathrm{~cm}^{-3}$ (Young et al. 2009). We have considered both the lines for our study. The Fe XIII $203.8 \AA$ line, too, is a combination of 203.79 and $203.82 \AA$ lines, with a component of Fe XII at $203.72 \AA$. Therefore, the total intensity under the Fe xIII line at $203.8 \AA$ is the total of $203.79+203.82 \AA$ intensities. Hence we have fitted two Gaussians to separate out Fe XII and Fe XIII lines. The two Fe XIv lines are not blended and have been fitted with single Gaussians.

\section{Data Analysis and Results}

\subsection{Formation and Evolution of TELs}

Fig. 2 displays the evolution of the two ARs between December 10-14, 2014 as observed with AIA using $193 \AA$ filter. The AR 12230 in the southern hemisphere first appeared on the visible side of the solar disk on 5th December 20143. However, it was identified as an AR and given its number on 7 th December 2014. The other AR 12234 located in the northern hemisphere was first detected on 10th December 2014. To show the evolution of the structures better, note that we have differentially rotated all the images displayed here at 19:35:30 UT on 12th December 2014 that corresponds to the center time of the EIS raster E2. Fig. 2 demonstrates that as early as on 10th December, the two ARs have individual loop structures along with a slight hint of faint TELs, connecting the two ARs. These TELs become prominent on 12th December (panel C), along with an X-shaped structure that is observed between $\mathrm{x}=\left[140^{\prime \prime}, 260^{\prime \prime}\right]$ and $\mathrm{y}=\left[-80^{\prime \prime},-20^{\prime \prime}\right]$ (see panels D, E and F). The X-shaped structure exists through 12th December. By 13th December, the ARs drift across the disk, towards the western limb, with gradual disorientation of the loops. The very well-defined morphology of the TELs are no longer visible on or after 14th December.

An animation tell2dec.gif created with data taken at a cadence of 30 minutes using the AIA $193 \AA$ channel images on 12th December, shows a continuous interaction amongst the loops belonging to the individual ARs as well as the TELs. On the one hand, we identify epochs when loops belonging to individual ARs interact and form TELs (e.g., refer to the frames at

\footnotetext{
1 EIS Software Note No. 16, P. Young, 2015

2 EIS Software Note No. 17, Peter Young, April 2013

3 as inferred from www.solarmonitor.org
} 


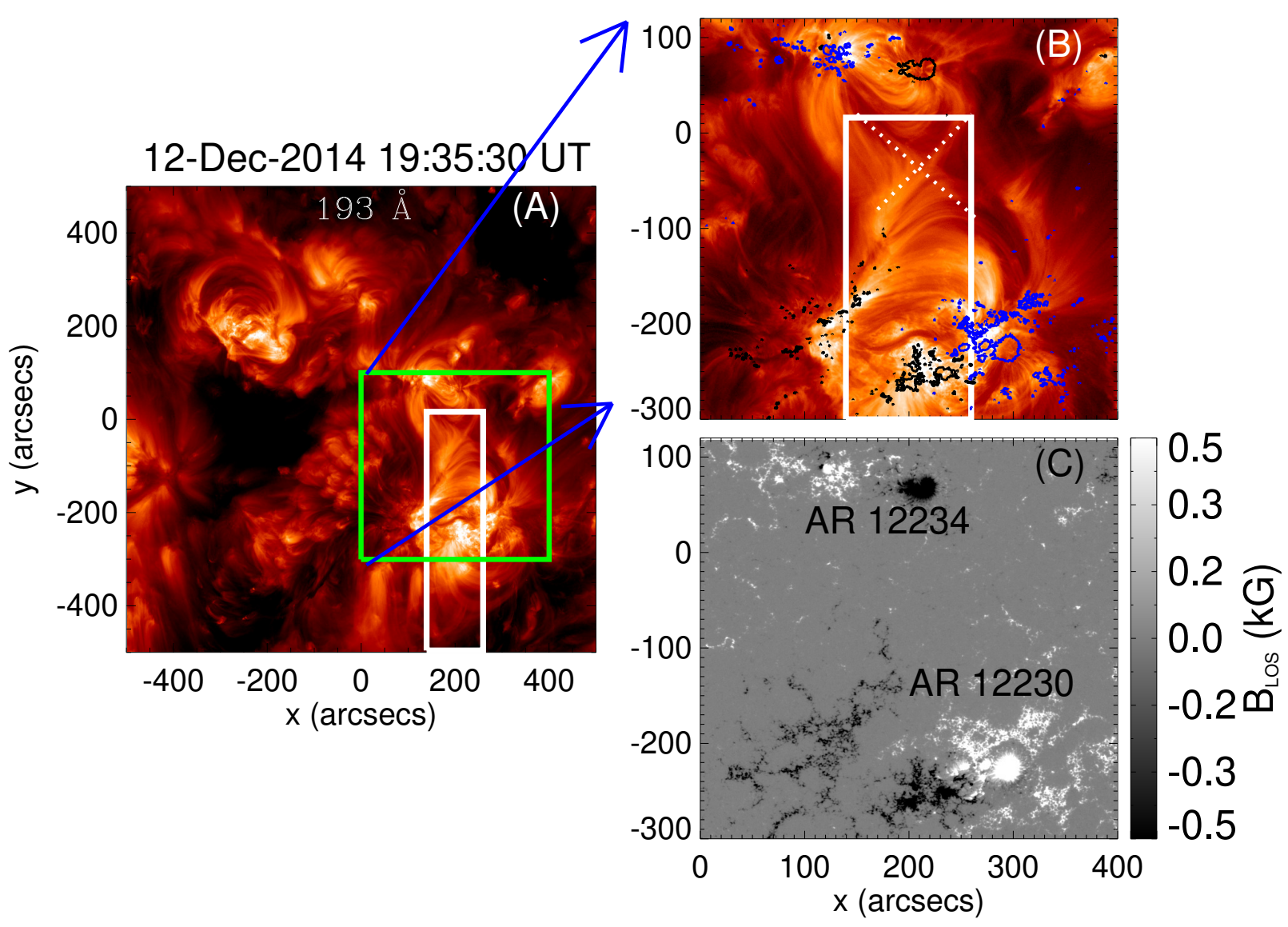

Fig. 1. Panel (A): AIA $193 \AA$ images showing the portion of the solar disk that contains the two active regions between which the trans-equatorial loops are formed. The over-plotted green box shows the FOV of AIA that is considered for further analysis. The white box is the FOV that was rastered using EIS. Panel (B): Zoom-in view of AIA $193 \AA \AA$ images corresponding to the green box in the left panel showing the X-type topology formed between the TELs (white dotted line). The white box shows the EIS raster FOV. The white dotted lines are drawn to guide the eye on the $\mathrm{X}$-shaped structure. The blue and black contours represent positive and negative polarity regions, respectively with magnetic flux density of $\pm 500 \mathrm{G}$ as observed by HMI at 19:35:40 UT. Panel (C): HMI LOS magnetogram image at the same instant as shown in the contours of panel B. The two active regions $A R 12230$ and $A R 12234$ are marked here.

13:00 hours in the animation). On the other hand, we also identify the interaction among the TELs leading to the reformation of loops belonging to their parent ARs. To highlight this, we show in panel A of Fig. 3 loops belonging to individual ARs (marked as 'I'), along with some TELs on the left of the FOV (marked as ' $\mathrm{T}$ '). In panel $\mathrm{B}$, we see the 'I' loops coming close enough so as to merge and form new TELs on the right of the X-region. Similarly, we see these TELs interacting amongst themselves in panel C. Finally, in panel D, we see such TELs have been annihilated to form more loops belonging to the two ARs.

\subsection{Loop dynamics}

To further understand the dynamics of the loops, we created xtplots along two artificial 10-pixels wide slits named ' $\mathrm{V}$ ' and ' $\mathrm{H}$ ' (see panel A in Fig. 4). For this purpose, we have used the data taken on December 12, 2014, with AIA $193 \AA$ at a cadence of 1 minute. Panels B and C of Fig. 4 show the xt-plots corresponding to the slits $\mathrm{V}$ and $\mathrm{H}$, respectively. The horizontal black dashed lines trace the locus of point $P$ labelled in panel A whereas the solid vertical black lines locate the four EIS raster phases, marked as E1, E2, E3 and E4.

The xt-plot corresponding to the slit V (panel B) demonstrates the inward movement of the structures from the very be- ginning and appear to merge soon after the raster E1, approximately between 12:00 and 13:00 UT. Such inward motion in the $\mathrm{xt}$-plot along the slit $\mathrm{V}$ suggests the evolution of loops belonging to individual active regions and moving closer to each other. During the same interval, the xt-plot corresponding to slit $\mathrm{H}$ (panel C) reveals hints of outward motion, though not as systematic as the case for the inward movement. The outward movement in the xt-plot for slit $\mathrm{H}$ suggests the structures are moving away. Combining the two xt-plots, we infer that loops belonging to the individual active regions are evolving. Their tips are coming closer to each other, leading to magnetic reconnection and formation of the TELs. We have highlighted the inward and outward motions with blue slanted (dashed-doted) lines in panels B and C.

After 13:00 UT, the motions observed in the two xt-plots reverse direction till about 19:00 UT. The reversing of movements suggests that during this period, the TELs are evolving and moving towards each other. During this time, the TELs reconnect and lead to the reformation of the loops belonging to individual active regions. Beyond 19:00 UT on December 12, 2014, the motion is similar to that is observed during the first phase.

We have used the over-plotted blue dashed-dotted lines in panel B (by eye estimation) to obtain the inflow speeds during the first and third phases. The inflow speeds are 1 and $3 \mathrm{~km} \mathrm{~s}^{-1}$, whereas the outflow speed for the second phase is $1.5 \mathrm{~km} \mathrm{~s}^{-1}$. 

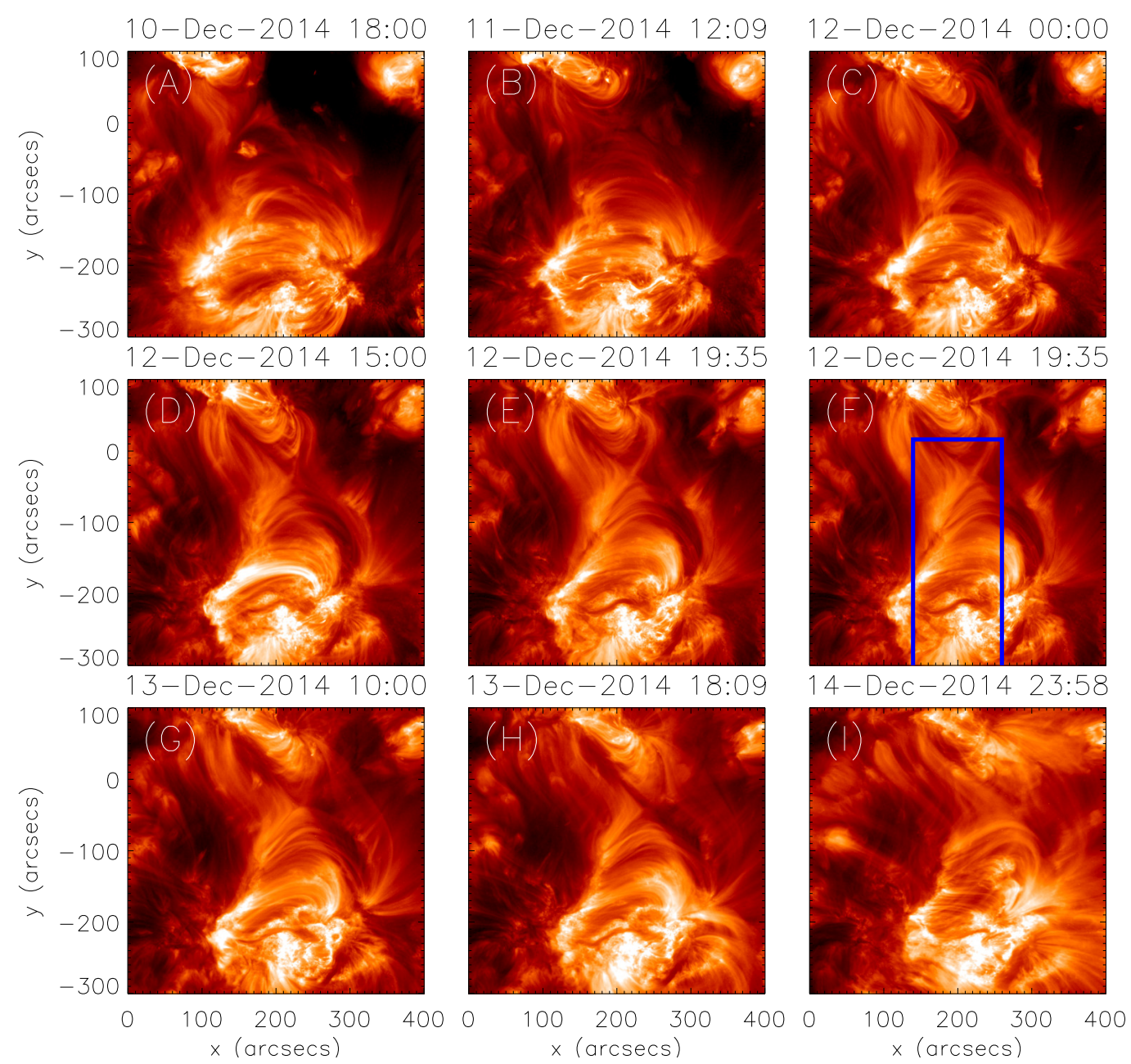

Fig. 2. Formation and evolution of the region showing TELs (corresponding to the FOV shown in panel (B) of Fig. 1) as observed in AIA $193 \AA$ images for five days from December 10-14, 2014. The over-plotted blue box in panel (F) shows the EIS raster FOV. All the AIA data shown here are differentially rotated to the center time of raster E2.

Due to ambiguous patterns observed in the xt-plot shown in panel C, we did not measure speeds along the slit H. Similar values of inflows and outflow speeds were also reported by Yokoyama et al. (2001) in an observation related to a flare.

\subsection{Identification of the reconnection point with AIA images}

As mentioned earlier, the X-region was rastered with EIS four times on 12 th December. Since the raster takes time to scan through the FOV, we identify a region that was simultaneously observed by AIA and EIS slits. For this purpose, we plot in Fig. 5 the AIA intensity maps in $193 \AA$ channel corresponding to 'E2' (panels A-F and $\mathrm{H}$ ). However, the ROI plotted in these maps is between $\left.\mathrm{y}=\left[-80^{\prime \prime}, 10^{\prime \prime}\right]\right)$ so as to highlight the dynamics/ features in the neighbourhood of the X-region. We identify intermittent brightenings occurring between $\mathrm{x}=\left[217^{\prime \prime}, 222^{\prime \prime}\right]$ and $\mathrm{y}=\left[-34^{\prime \prime}\right.$, $\left.-29^{\prime \prime}\right]$, which is henceforth identified as 'P2' (marked with the yellow box). Coincidentally, this region is also observed by four EIS raster exposures (highlighted by the white vertical lines in panel C).

In panel $\mathrm{G}$ (just beneath panel $\mathrm{C}$ ), we plot the average light curve in this box with a cadence of 12 seconds between 18:55:07-20:14:55 UT. The bold black vertical lines represent the four exposures of E2 raster, each $2^{\prime \prime}$ wide, corresponding to the white lines marked in panel $\mathrm{C}$. In addition, the exact duration of E2 is marked by the dashed black vertical lines in panel $\mathrm{G}$. From the light curve, we see that three intensity peaks occur in quick succession at $\mathrm{P} 2$. This indicates dynamic events occurring and are also captured by simultaneous EIS raster observations. This gives us the opportunity for measuring the plasma parameters in the P2 region. Similarly, for E4 observations, there is a partial overlap of EIS observations with the region having repeated intensity enhancement. However, no such spatial overlap of EIS exposures with those of homologous intensity enhancement is observed for E1 and E3.

\subsection{Plasma Diagnostics with EIS}

As stated earlier and shown in Fig. 1, the EIS spectrometer observed the X-shaped region four times on 12th December 2014, in raster mode. These are denoted as E1, E2, E3 and E4. We plot the EIS intensity maps obtained in different ionisation states of Iron from Fe vIII to Fe xv in Figs. 6 \& 7 (as labelled). In these maps, we emphasize the region between $y=\left[-120^{\prime \prime}, 10^{\prime \prime}\right]$, so as to show the morphology of the loops near the X-region as well as the loops beneath and above it, in detail. The four columns in these two figures correspond to the four different EIS rasters. We have differentially rotated the FOVs of E2, E3 and E4 to the raster start time of E1, to facilitate the time evolution study of 

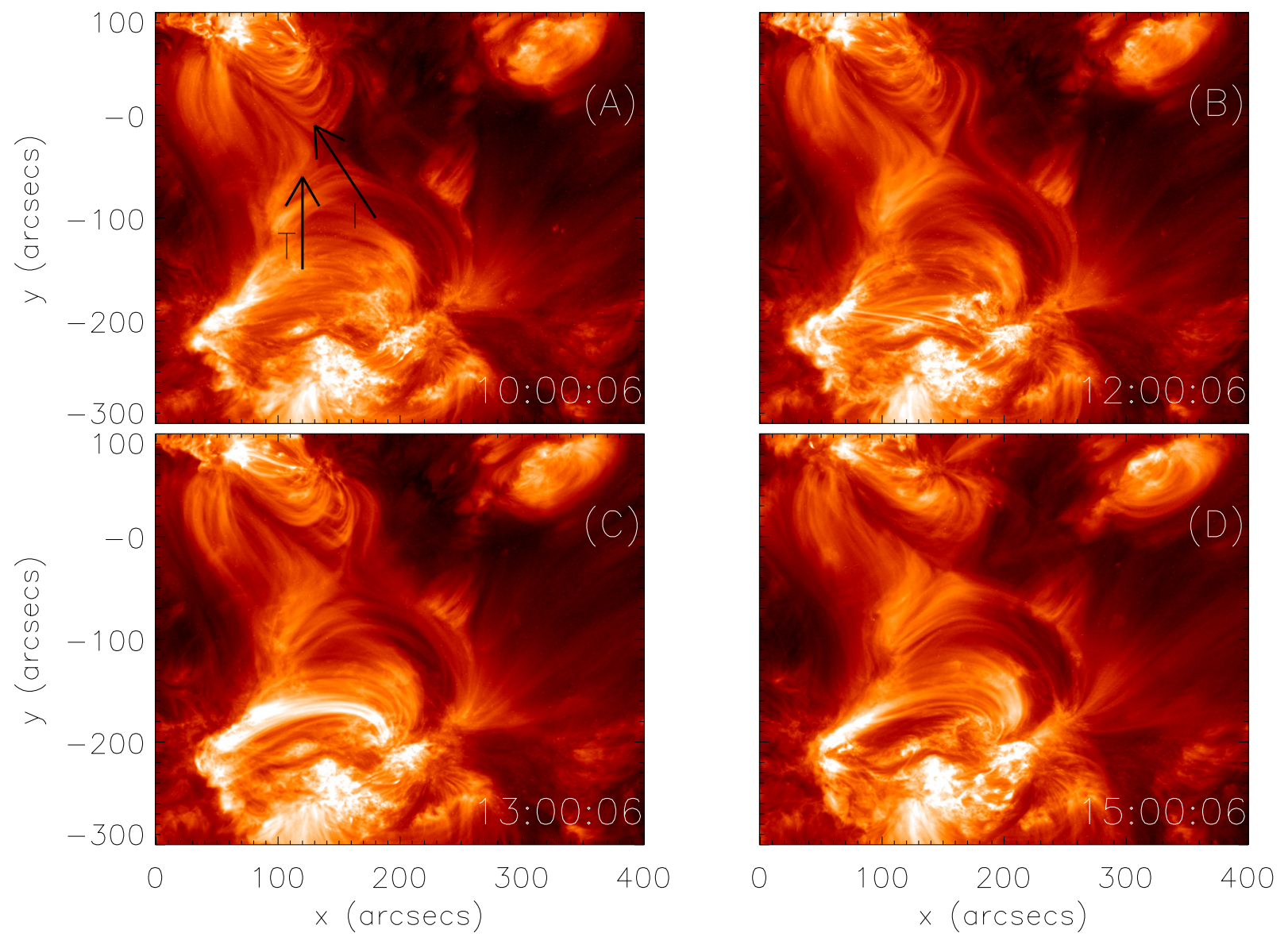

Fig. 3. Snapshots of events on December 12, 2014 showing formation of TELs and reformation of the loops belonging to individual ARs as a consequence of successive reconnections, as observed by AIA $193 \AA$ channel. For more details, please see the animation tel12dec.gif.

the same region. The noticeable rightmost black stripes in $\mathrm{E} 2$, $\mathrm{E} 3$ and $\mathrm{E} 4$ are due to this rotation. We locate the X-shaped region in all the intensity maps with a blue box, that encloses the point ' $\mathrm{P}$ ' shown in panel A of Fig. 4 .

The intensity maps obtained at cooler temperatures, such as in $\mathrm{Fe}$ VIII and $\mathrm{Fe} \mathrm{x}$, show a number of distinct loops criss-crossing each other (top and middle rows in Fig. 6). Similar structures are also seen in the intensity images obtained in Fe xI (bottom row of Fig. 6) and Fe XII (top row of Fig. 7) but with a significant amount of diffuse emission, similar to those observed by, for $e . g$., Tripathi et al. (2009); Subramanian et al. (2014). In the intensity images obtained at higher temperatures (exceeding $\log T[K]=$ 6.20 ), we notice a decrease in the intensity in the X-shaped region. The region appears fully evacuated in the intensity maps of $\mathrm{Fe}$ XIV and Fe XV for all the three rasters but E1 (bottom row of Fig. 7). It is worth noticing that there is an abundance of bright loops in the Fe xv intensity maps beneath the X-region.

To illustrate this better, we obtain light curves in five spectral lines of Iron for all four EIS rasters and plot them in Fig. 8 as labelled. The top panels are the intensity maps in Fe $\mathrm{Xv}$ and bottom panels are the light curves. We obtain the light curves by averaging the intensities within the two horizontal white lines overplotted in the top panels. We have drawn the vertical blue and black lines in the top and bottom panels, respectively, to highlight the extent (in $\mathrm{x}$-direction) of the $\mathrm{X}$-shaped region and the corresponding extent in the respective light curves.

The light curves (shown in the bottom panels of Fig. 8p reveal that the $\mathrm{X}$-shaped region is dimmer in all the spectral lines in all rasters, except E1, where Fe $\mathrm{xv}$ is brighter than other lines. However, it shows a decrease in intensity where Fe $\mathrm{x}$ shows an enhancement. Similar nature of light curves is noted for Fe XIV (not shown here), albeit it is a weak line and has minor fluctuations in intensity as a function of position.

\subsubsection{Density and Temperature diagnostics}

Plasma diagnostics are conducted in several regions within the EIS raster FOV, to discern the properties in different types of loop structures captured in the same. The average electron densities and temperatures have been estimated at the X-region along with those in the adjacent loops for all four rasters. These are denoted in Fig. 9 by 'P' (X-region), 'L' (TELs) and 'D' (hot loops beneath the X-region) on the Fe XII $195 \AA$ intensity map of $\mathrm{E} 2$, rotated with respect to $\mathrm{E} 1$. In addition, for $\mathrm{E} 2$, we have the fourth region of interest $\mathrm{P} 2$ which shows intermittent intensity enhancements, as shown in panel G of Fig. 5 .

\subsubsection{Assessment of background/foreground}

Estimate of background/ foreground emission plays an important role in measuring plasma electron densities and temperatures (Del Zanna \& Mason 2003; Tripathi et al. 2011). For this, we identify a region marked as ' $\mathrm{B}$ ' in Fig. 9 The average intensity in ' $\mathrm{B}$ ' is assumed to be the background/ foreground intensity. The green box in Fig. 9 is the zoomed-in ROI and correspond to the ROI that is shown in Figs. 6 and 7. 


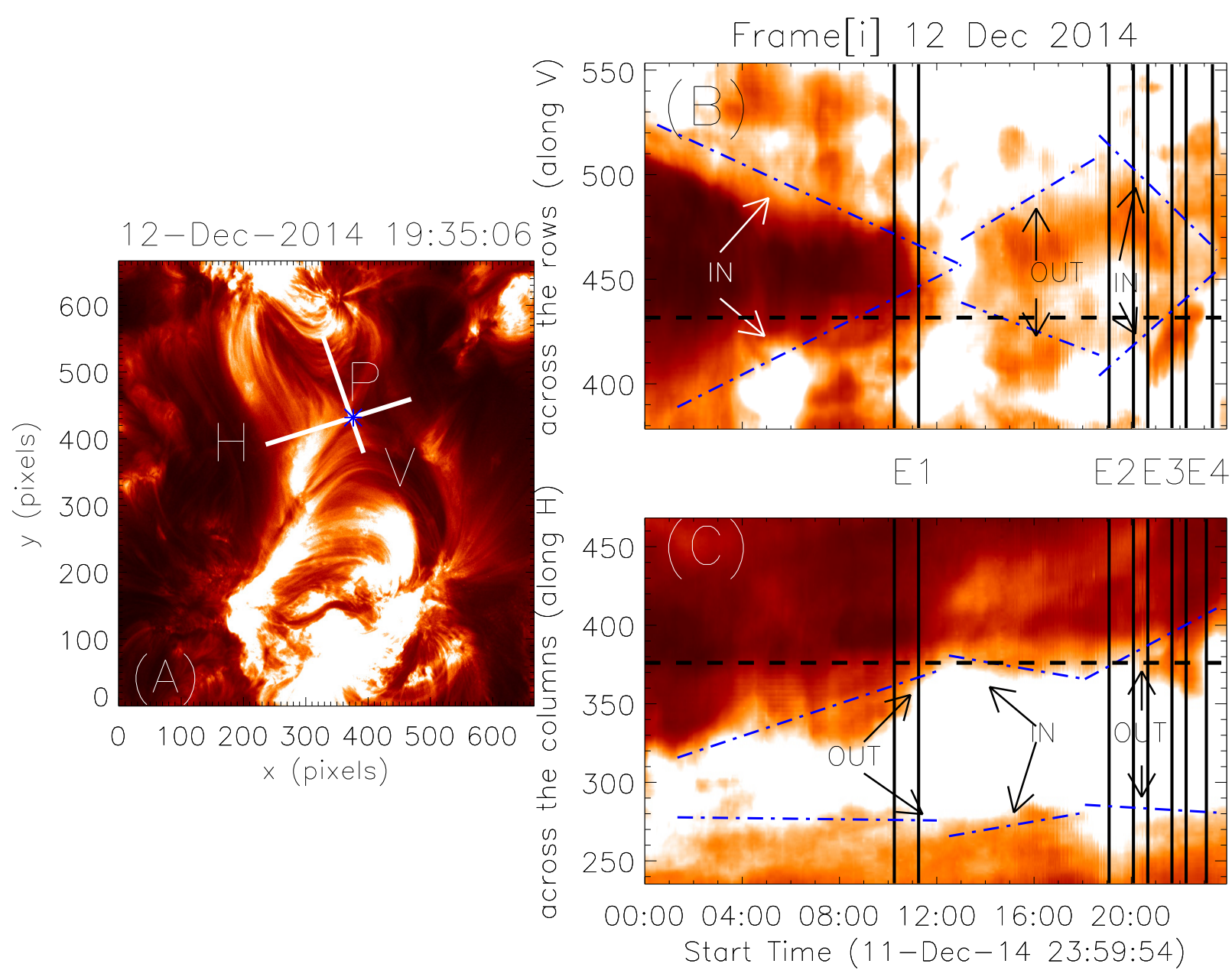

Fig. 4. Panel (A): The TELs seen in AIA $193 \AA$ A channel in pixel coordinates at a given instant belonging to EIS raster E2. The white lines are the slits, marked as ' $\mathrm{H}$ ' and ' $\mathrm{V}$ ' and the point ' $\mathrm{P}$ ' ('*', in blue) denotes the intersection. Panels (B) and (C): The intensity fluctuations along the slits ' $\mathrm{V}$ ' and ' $\mathrm{H}$ ' respectively in the xt plots. The over-plotted solid black lines indicate the EIS raster durations, with numbers 'E1', 'E2', 'E3' and 'E4' indicating the respective raster number. The dashed-dotted blue lines track the displacement of plasma with time. The black dashed line shows the position of the point ' $\mathrm{P}$ ' in pixel coordinates.

The averaged intensities for locations P, P2, L, D and B are noted in Table 2 . We note that the averaged intensities in Mg vII and $\mathrm{Si}$ VII in 'B' region larger than the actual locations which are considered for the estimates. Similar discrepancy is noted for Fe xIv line. We attribute this to the weak spectral lines and therefore, discard these three lines for any kind of plasma diagnostic studies. In addition, we note that for different locations for different rasters, the average intensities in ' $\mathrm{B}$ ' exceed those in the other three regions. This renders most of the low temperature lines viz., Fe viII, Fe Ix, Fex and Fe xI unsuitable for the EM-loci computation. That leaves only a set of four lines Fe XII $192 \AA$ and $195 \AA$, Fe xIII and Fe xv only. Therefore, we neglect the background/foreground correction for EM-loci plots for all three (four) regions, 'P', 'L', and 'D' (and 'P2' in case of E2).

\subsubsection{Density diagnostics in the $\mathrm{X}$-region}

Using the average intensities in ' $\mathrm{B}$ ' in Fig. 9 as the background/ foreground values, we derive the density maps for Fe XII and Fe xIII line pairs (refer to Fig. 10p for all four raster periods. Note that these maps show background subtracted densities. The four columns represent the four different rasters as labelled. The overplotted white-box in each map highlights the X-shaped structure (same location as the blue boxes in Figs. 6 \& 7). It is further noted that these boxes enclose the point ' $\mathrm{P}$ ', shown in panel A of Fig. 4 . We neglect deriving density maps obtained using Fe xI and Fe XIV, since these are noisy. This is because of one of the lines of Fe XI, i.e. 180.401 $\AA$ is at the edge of CCD A where the effective aperture area is small; and Fe xIv lines have small signal to noise ratios as they are weak.

The average densities within the $\mathrm{X}$-shaped regions (enclosed by white boxes) for all four rasters using the Fe XII (similar for Fe xIII) lines range between $\log N_{e}=8.46$ to $8.67 \mathrm{~cm}^{-3}$ (also see, Table 3). To check the consistency of our results, we compared these densities by obtaining the averaged spectra within the white box before taking the ratios. The density estimates were similar to those listed in Table 3 In addition, the density at the identified brightening observed by EIS slits, i.e., $\mathrm{P} 2$ is $\log N_{e}$ $=8.50$ in Fe xII, which is consistent with the average values in the X-region.

\subsubsection{Temperature diagnostics in the X-region}

We estimate the temperature of the $\mathrm{X}$-shaped region, which is shown by blue boxes in Figs. 6 \& 7 7 , using the Emission Measure (EM) loci curves (Jordan \& Wilson 1971; Tripathi et al. 

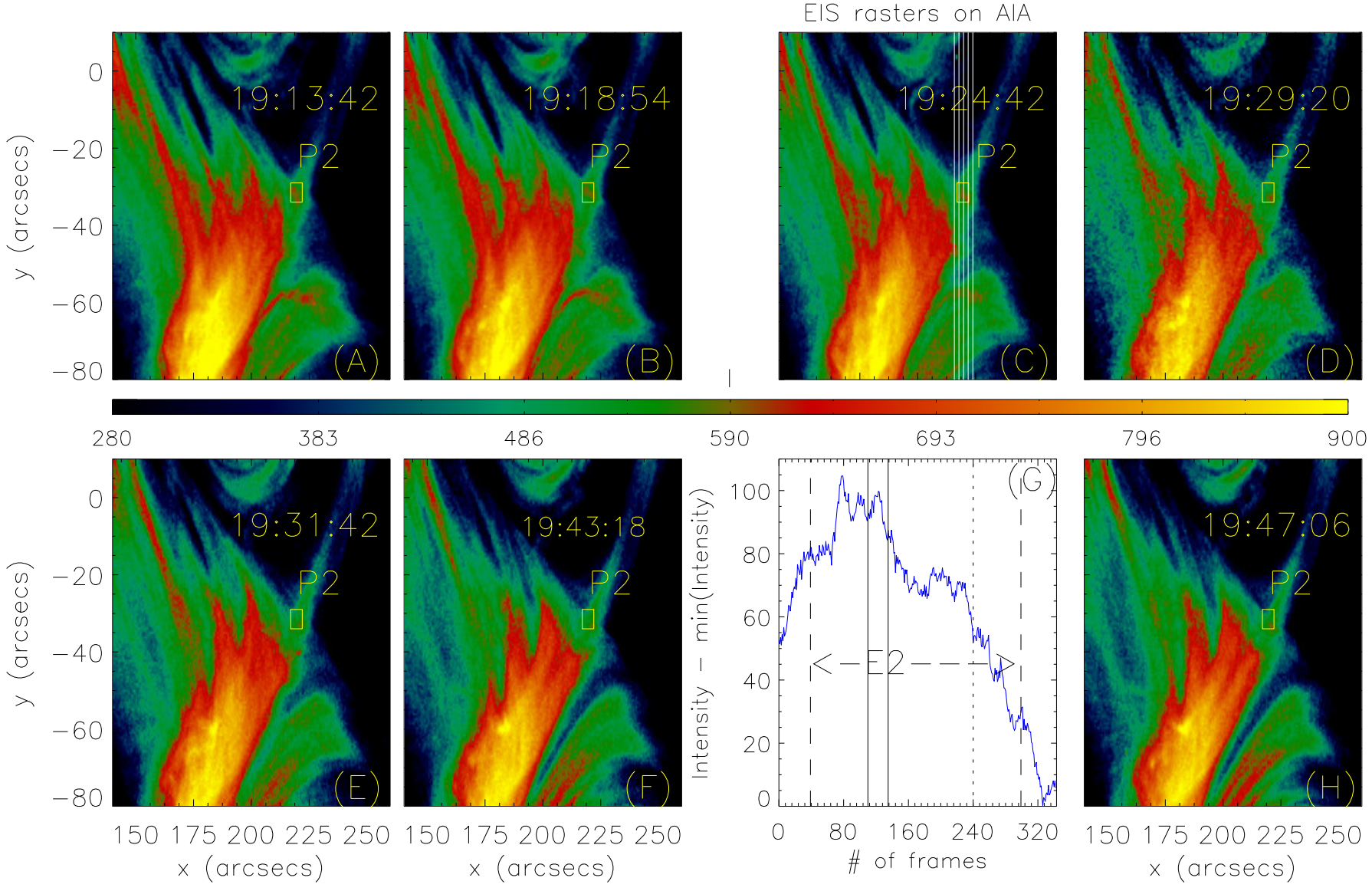

Fig. 5. Panels (A)-(F) \& (H): AIA $193 \AA$ intensity images at different instants, roughly coinciding with the EIS observation period for E2. 'P2' (highlighted by the yellow box) denotes the location for recurrent brightenings. The white vertical lines in panel (C) show that four EIS exposures which cover the location of this brightening. Panel $(\mathrm{G})$ : The corresponding light curve in 'P2', between 18:55:07-20:14:55 UT at a cadence of 12 seconds. The exact duration of E2 is marked by the dashed vertical black lines. The black bold lines indicate the increase in intensity in 'P2' corresponding to the EIS exposures shown with white lines in panel C.

2010). For this purpose, we first obtain the averaged intensities in the region in all the spectral lines marked with ' $t$ ' in Table 1 . However, we exclude Si viI, for reasons explained in $\$ 3.4 .1 .1$. To compute the EM we have used coronal abundances of $[$ Schmelz et al. (2012) and standard ionization equilibrium given by CHIANTI (Dere et al. 1996; Del Zanna et al. 2015). The obtained EM-loci curves for all four rasters in the X-region are shown in Fig. 11. We have also plotted the histograms of number of crossings of EM curves within a temperature bin of $\log T[K]=0.1$ in the respective panels. In all the four plots, the left $y$-axis denote the EM values, whereas the right $y$-axis represents number of crossings in each temperature bin.

The EM-loci curves along with the histograms suggest that the plasma within the $\mathrm{X}$-shaped structure (enclosing the point $\mathrm{P})$ during E2 is nearly isothermal with temperature within $\log T[K]=6.10-6.20$. In contrast, for E3 we note that the plasma is more multi-thermal in nature, with the histogram peaking between $\log T[K]=6.10-6.30$. The average temperatures in the X-region for all four rasters are noted in in Table 3 .

For the P2-region, the EM-loci curves are shown in Fig. 12 It reveals that the peak formation temperature ranges between $\log T[K]=6.20-6.30$ (refer to Table 3), which is somewhat larger than that noted in the X-region.
3.4.1.4. Plasma density and temperature diagnostics in the adjacent loops

It is imperative to have a comparison of the electron densities and temperatures in the $\mathrm{X}$-region (the point $\mathrm{P}$ is contained in this region) with those at the adjacent loops. Therefore, in Fig. 9 we have identified two additional regions- one on the TELs (shown in blue and identified as ' $L$ ') and other at the loops belonging to the active region in the southern hemisphere (shown in black, identified as ' $D$ ') of the X-region. The background is Fe XII $195 \AA$ intensity map corresponding to E2. The average densities obtained at L, in most cases, is higher than those obtained at P. The former, in turn, is always less than those in D (see Table 3). We note that densities in the TELs obtained here using Fe XII lines is about an order of magnitude lower than those obtained by Liu et al.(2011) using the line ratios of Si x observed by CDS.

In Fig. 13, we also plot the EM Loci curves obtained for locations $\mathrm{L}$ and $\mathrm{D}$ for E2 raster period. The plots show that the plasma at location D is more multi-thermal than location L. We further note that the temperature for TELs and loops corresponding to the active region in the southern hemisphere are somewhat higher (and more multi-thermal) than those obtained P i.e., within the X-shaped region (see Table 3). The temperatures ob- 

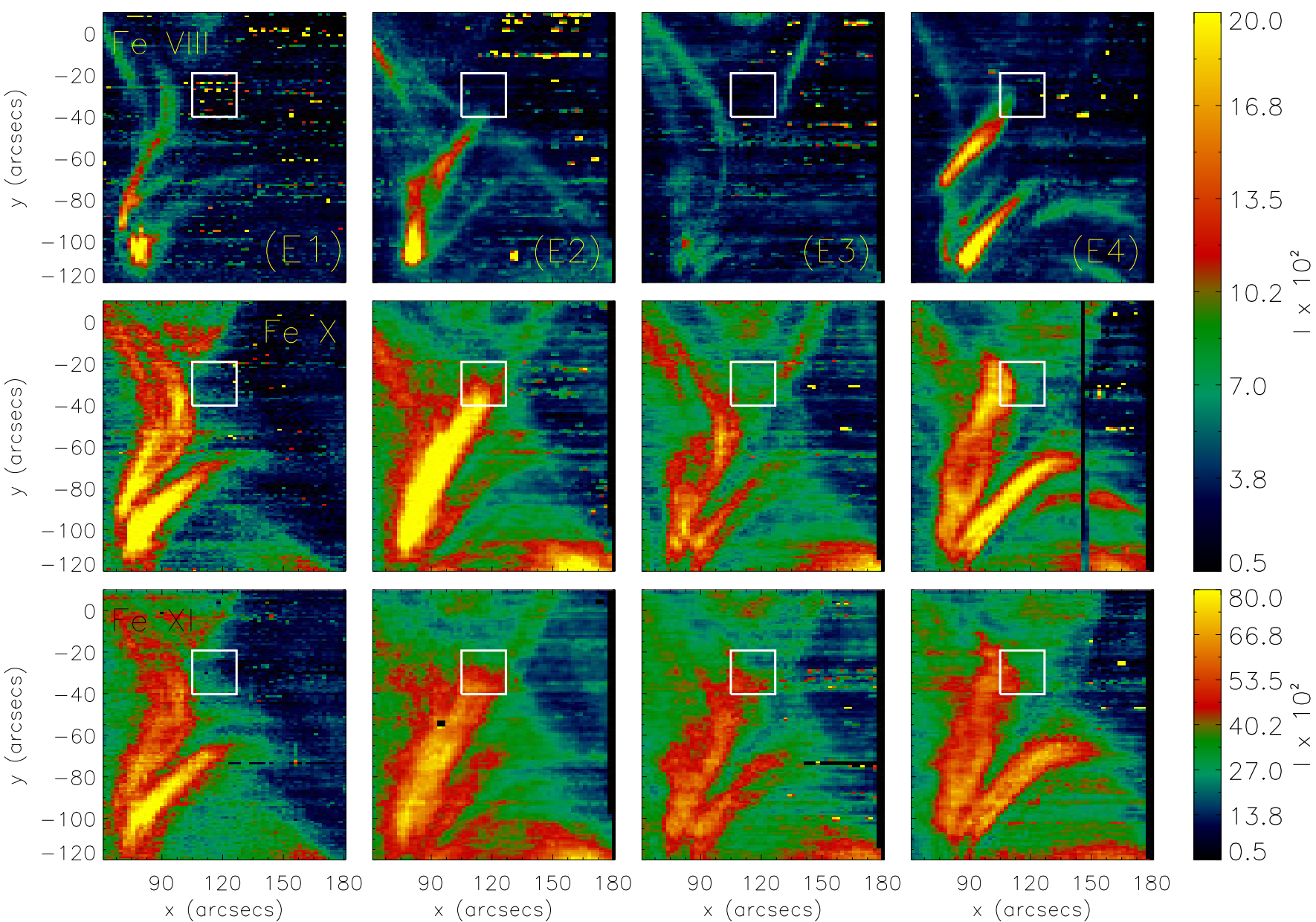

Fig. 6. The intensities in EIS Fe viII, Fex and Fe xI lines for the four events. The FOV of E2, E3 and E4 have been rotated to that of E1. The white box shows the reconnection region. The raster number on the top panel of each column is true for all the spectral lines whereas the each row corresponds to the same spectral line as mentioned in the leftmost panel of each row.

tained for locations $\mathrm{L}$ and $\mathrm{D}$ are in agreement with those obtained for TELs by Sheeley et al. (1975); Delannée \& Aulanier (1999); Glover et al. (2003); Pevtsov (2004); Balasubramaniam et al. (2005). This temperature range is maintained throughout the four rasters.

\subsubsection{Doppler velocities and Spectral Line Width}

We obtain the Doppler velocity and line width maps corresponding to the X-region for all four rasters in Fe XII, Fe XIII and Fe Xv. The maps are displayed in Figs. 14 \& 15 The four columns represent the four EIS rasters. The over-plotted white (black) boxes in Doppler (line width) maps correspond to the blue boxes in Figs. 6 \& 7, enclosing the X-shaped structure.

The Doppler velocity maps for Fe XII and Fe XIII are very similar. During the second raster E2, when the region is closest to the disk center, the $\mathrm{X}$-shaped region shows dominant redshifts of $\sim 5-8 \mathrm{~km} \mathrm{~s}^{-1}$. On the contrary, during rasters E1 and E3, the same region shows predominant blueshifts of $\sim 10 \mathrm{~km} \mathrm{~s}^{-1}$. For the raster E4 when the active regions is farthest from the disk center, the LOS velocities are predominantly upflows $\left(\sim 5-8 \mathrm{~km} \mathrm{~s}^{-1}\right)$. At higher temperature mapped by Fe xv line, a significant fraction of the pixels is observed to have blueshifts (E1), redshifts (E2, E3) and very close to zero (E4) velocities. The average Doppler velocities in the box region are noted in Table 3 Taking into ac- count an uncertainty of $\sim 5 \mathrm{~km} \mathrm{~s}^{-1}$ in EIS velocity measurements (see e.g., Young et al. 2012), we note that the direction of plasma flows along the LOS do not change.

The line width maps shown in Fig. 15 are essentially the FullWidth-Half-Maxima (FWHM) obtained after subtracting the in-

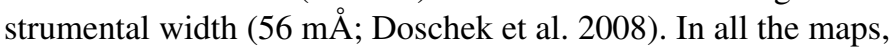
the loops at the bottom have relatively higher FWHMs, but are best identified in Fe xII. The FWHMs obtained within the box is $\sim 0.03 \AA$ for Fe XII and Fe XIII lines, for all four rasters (see, Table 3), which is equivalent to the non-thermal velocity of $\sim 27 \mathrm{~km} \mathrm{~s}^{-1}$. To derive the non-thermal velocities, we have used the temperature in the X-region to be around 1.6 MK $(\log T[K]=6.20)$ as was obtained using the EM-Loci procedure in 3.4.1.3. However, there are a few individual pixels with FWHM as large as $0.068 \AA$ with an equivalent non-thermal velocity of $\sim 62 \mathrm{~km} \mathrm{~s}^{-1}$.

The Fexv line has an average FWHM of $\sim 0.048 \AA$ $\left(\sim 30 \mathrm{~km} \mathrm{~s}^{-1}\right)$ in X-shaped structure for all four rasters. There are pixels within the box, where the FWHM is as large as $0.18 \AA$, which is equivalent to $115 \mathrm{~km} \mathrm{~s}^{-1}$ (assuming $\log T[K]=6.2$ ).

The average Doppler velocity and non-thermal width in P2 region are also noted in Table 3 and seen to be fairly similar to those in the X-region (within the error limits). In addition, the other plasma parameters in P2 (averages) are similar to those 

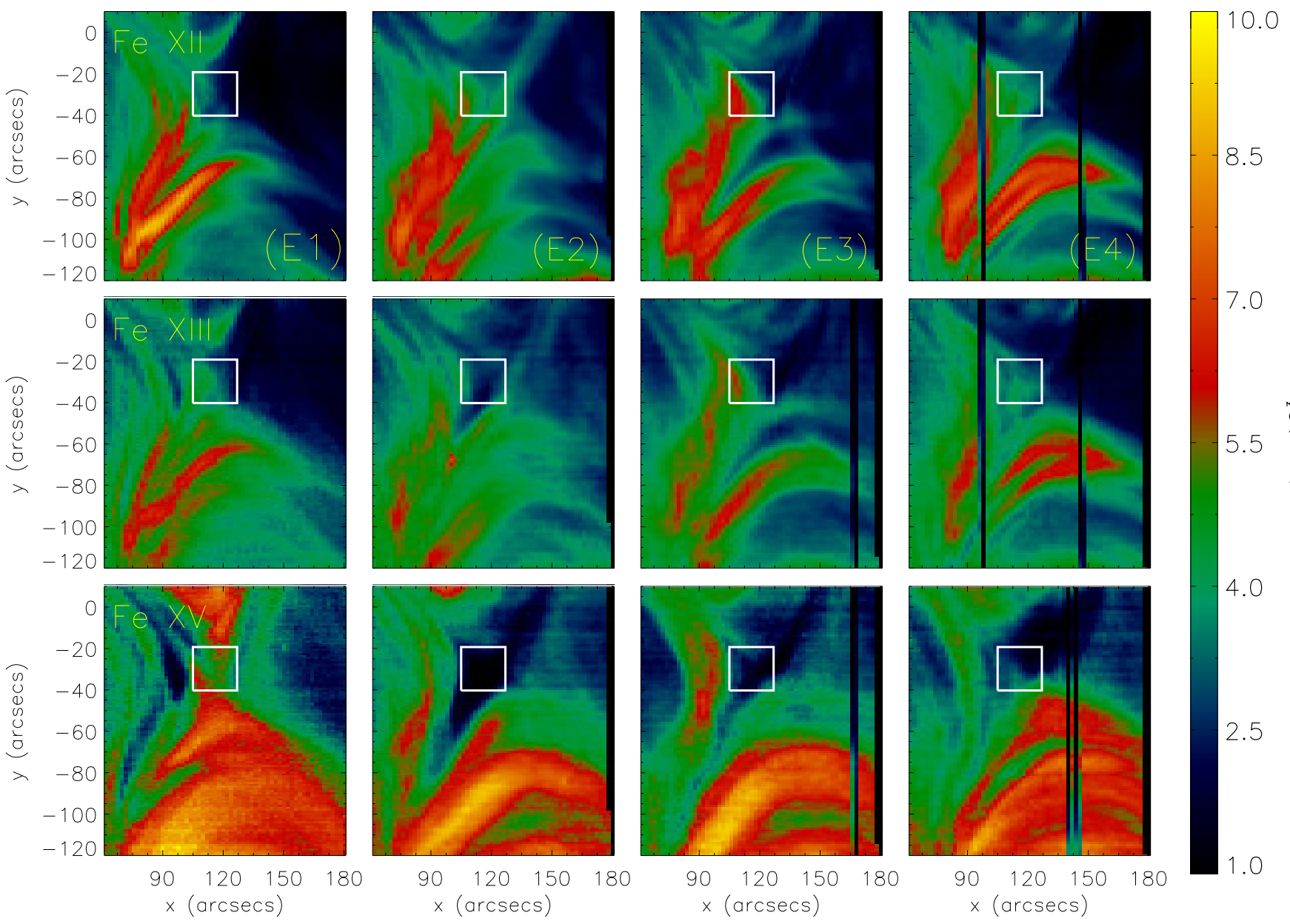

Fig. 7. Same as Fig. 6 but for Fe xII, Fe XIII and Fe xv lines.
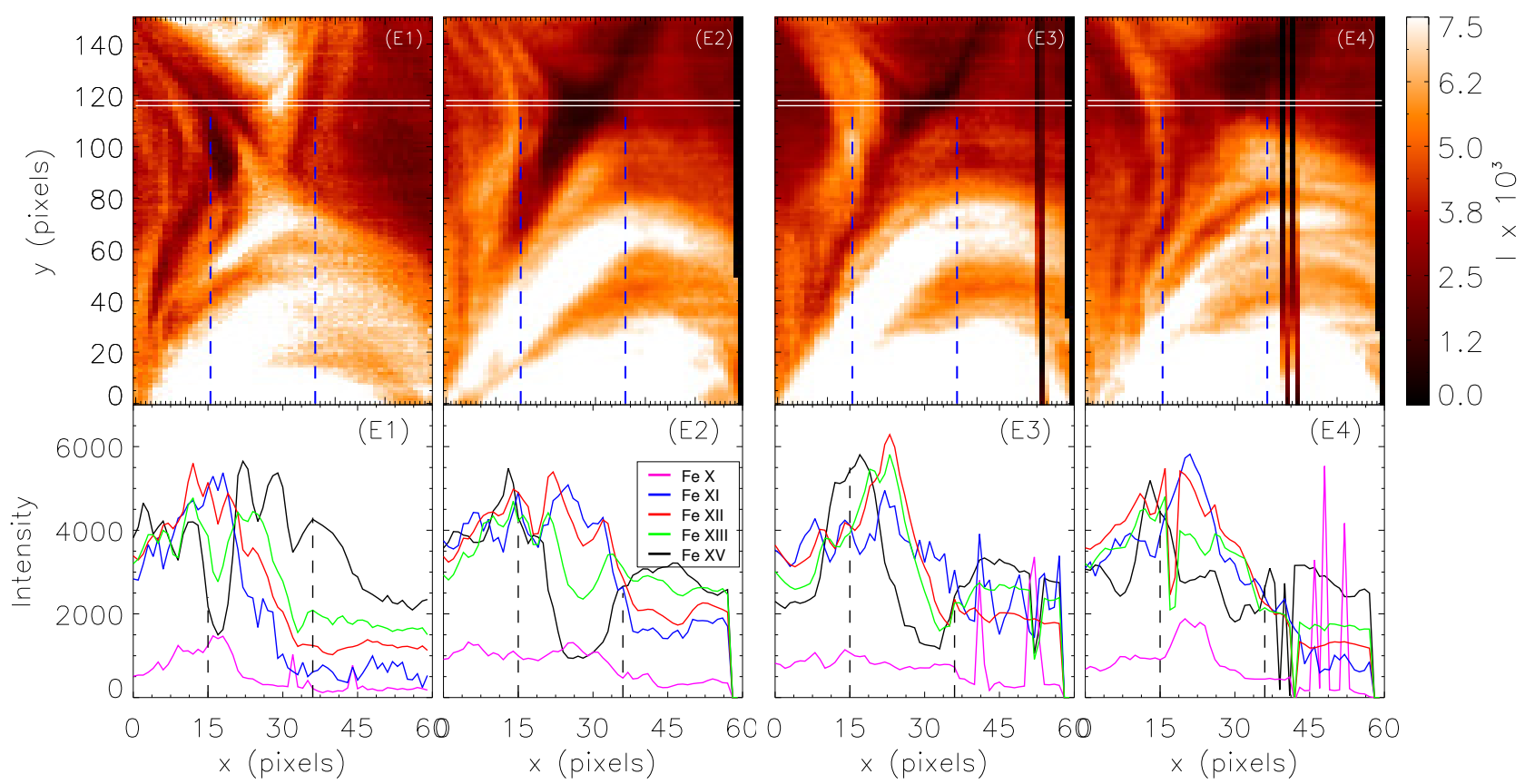

Fig. 8. Top panels: EIS intensity maps obtained in Fe xv. The horizontal white stripe is the region for which the averaged light curve is obtained. Bottom panels: Average light curves within the white stripes in spectral lines as labelled. The vertical black dashed lines in the bottom panel(s) correspond to the same pixel position as indicated by the vertical blue dashed lines in the top panel(s), highlighting the intensity variation in the X-region. 


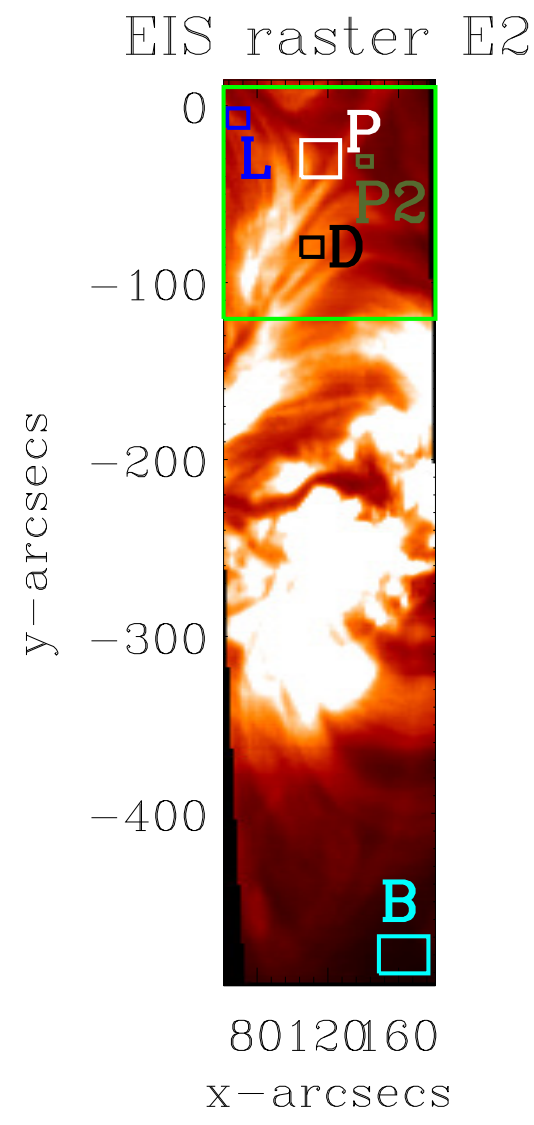

Fig. 9. Identification of different regions in the FOV for the TELs ('L', in blue), X-shaped region ('P', in white) and the hot loops beneath ('D', in black) on the background intensity map of Fe XII $195 \AA$ at E2. 'P2' (in olive) is the region which undergoes several brightenings at regular intervals within the EIS observation period E2 and has four EIS exposures, coinciding exactly with the position of the brightening. The box identified with ' $\mathrm{B}$ ' marks the region considered for background/ foreground assessment. The large green box indicates the ROI of the study.
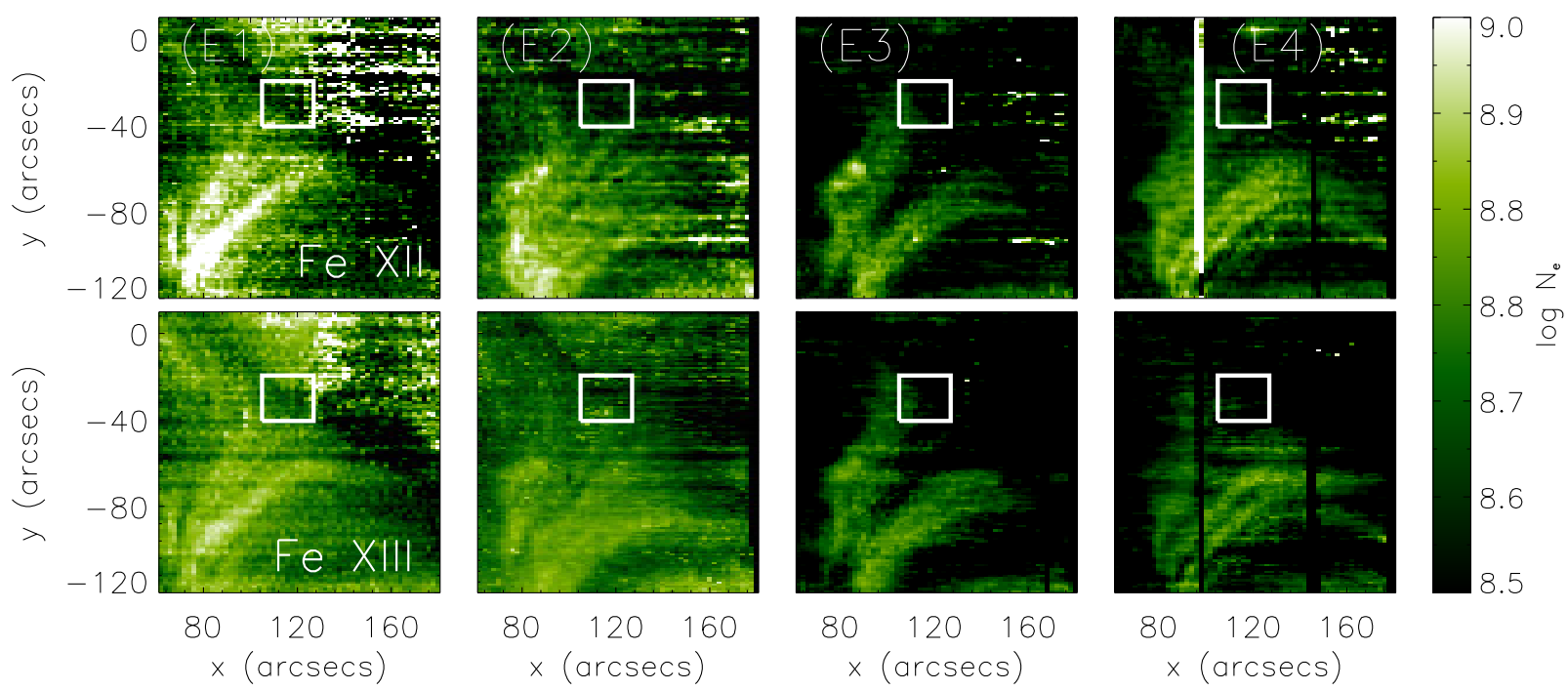

Fig. 10. Density maps using EIS lines Fe XII $(\log T[K]=6.20)$ and Fe XIII $(\log T[K]=6.25)$. The raster number on the top panel of each column is true for all the spectral lines whereas the each row corresponds to the same spectral line as mentioned in the leftmost panel of each row. The white box in each panel shows the X-region. Backfround/ foreground emission has been accounted for.

in the X-region. Therefore, we conclude that the X-region well represents the reconnection region, for all events. 

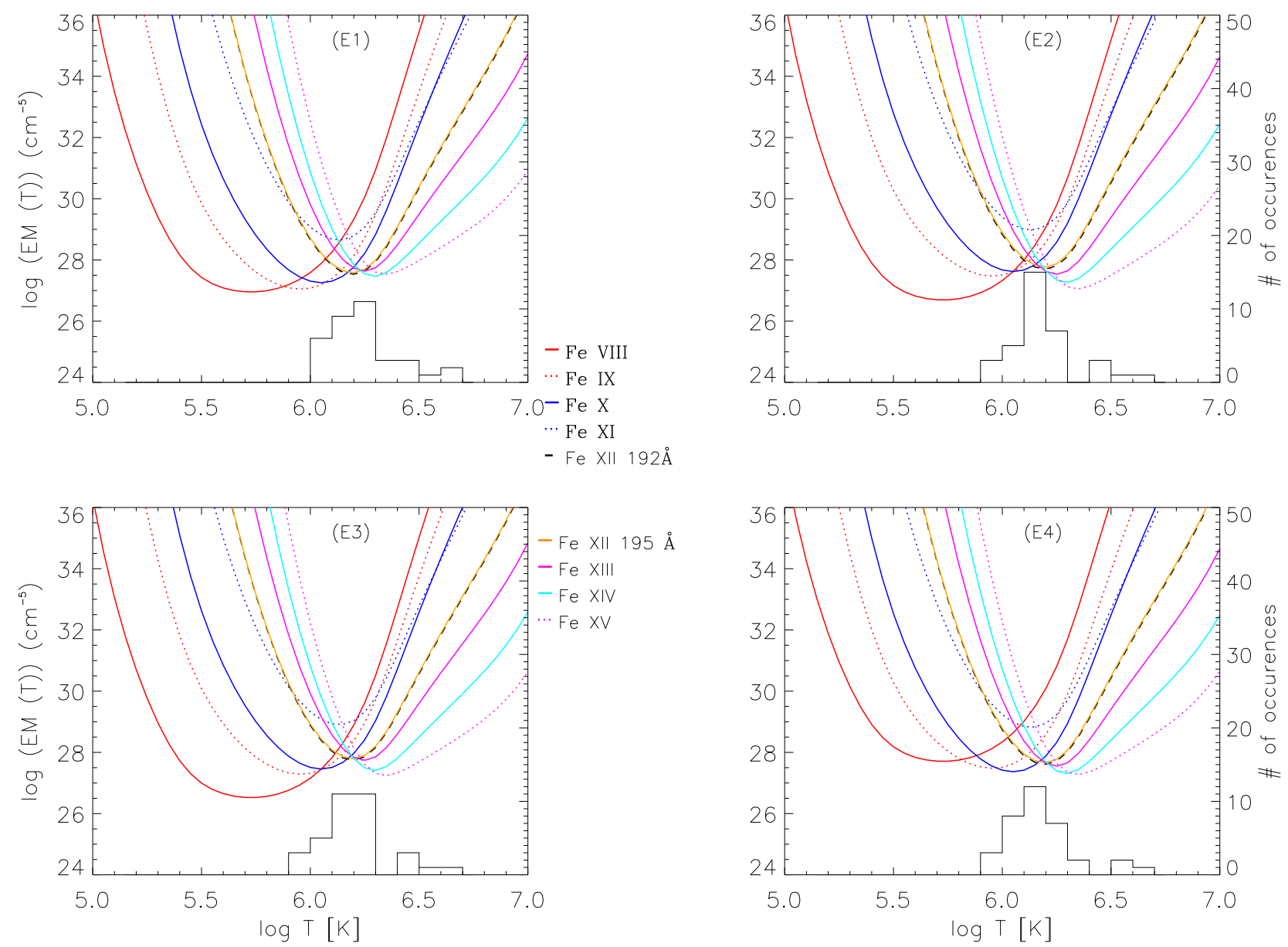

Fig. 11. Emission Measure (EM) loci curves for the four EIS rasters for the X-region as shown in Fig. 10 Also plotted are the histograms representing number of crossings within a given temperature bin. No background/ foreground emission has been considered.

\section{Summary and Conclusions}

This piece of study combines observations recorded by the AIA and the HMI on-board SDO and EIS on-board Hinode to study the formation, dynamics and plasma parameters of the transequatorial loops (TELs). The TELs were observed between preexisting $A R 12230$ in the southern hemisphere and the newly emerging $A R 12234$ in the northern hemisphere. We have performed a comprehensive study of the evolution of TELs using AIA observations. The physical plasma parameters such as density, temperature, Doppler and non-thermal velocities in the loops as well as the interaction region between those loops are studied using EIS spectroscopic observations.

The observations recorded by AIA reveal that initially, loops belonging to the individual ARs evolve. These loops come closer to each other and form the X-shaped topology, leading to the formation of the TELs. This is highlighted in Fig. 3 and is suggestive of the occurrence of the process of magnetic reconnection. The xt-plots in Fig. 4 show that the inflow speed for the reconnection is $1-2 \mathrm{~km} \mathrm{~s}^{-1}$ whereas the outflow speed is $\sim 2 \mathrm{~km} \mathrm{~s}^{-1}$. These values are in close comparison to that of Yokoyama et al. (2001); Liu et al. (2011) in a flaring TEL system $\left(\sim 5 \mathrm{~km} \mathrm{~s}^{-1}\right)$. However, we note that these are projected velocities measured in the plane-of-the-sky, hence gives the lower limit on the estimates.

The intensity maps corresponding to the four EIS raster periods are derived using various spectral lines formed between $\log T[K]=5.80-6.35$. At lower temperatures, corresponding to Fe vIII to Fe $x$, the X-region is seen very clearly with the adjacent loops being very bright. The intensity images of Fe XII (formed at $\log T[K] \leq 6.20$ ), show bright X-regions whereas at Fe Xv $(\log T[K]=6.35)$, the X-region appears to be filled with plasma at E1 but completely void at E2 and partially filled at E3 and E4. Such darkening at the reconnection site has been attributed to density diminution (Delannée \& Aulanier 1999, Tripathi et al. 2007, Sun et al. 2015). This result is reinforced by plotting the light curves in Fig. 8 Yokoyama et al. (2001) reported similar voids in soft X-ray observations of magnetic reconnection events leading to flares.

Within the X-region for all the four EIS raster periods, the electron densities are maintained steadily at $\log N_{e}=8.46$ to $8.67 \mathrm{~cm}^{-3}$ for the Fe XII and Fe XIII lines. The EM-Loci curves suggest that the plasma is very nearly isothermal at $\log T[K]=$ 6.20 (i.e. $1.6 \mathrm{MK}$ ) within the X-region, which is somewhat larger than those reported by Liu et al. (2011) at the cusp region (1.3 MK). However, Sun et al. (2015) found higher temperatures, between $1-5 \mathrm{MK}$ at the magnetic reconnection site between TELs, but with an associated flare. Also, there are hints of multithermality of plasma in the X-region at E3.

High cadence observations, at an interval of 12 seconds, further, reveal intermittent brightenings occurring close to the $\mathrm{X}$ region (marked as P2 in Fig. 57. Coincidentally, such brightenings occurring during E2 phase of EIS raster observation were 


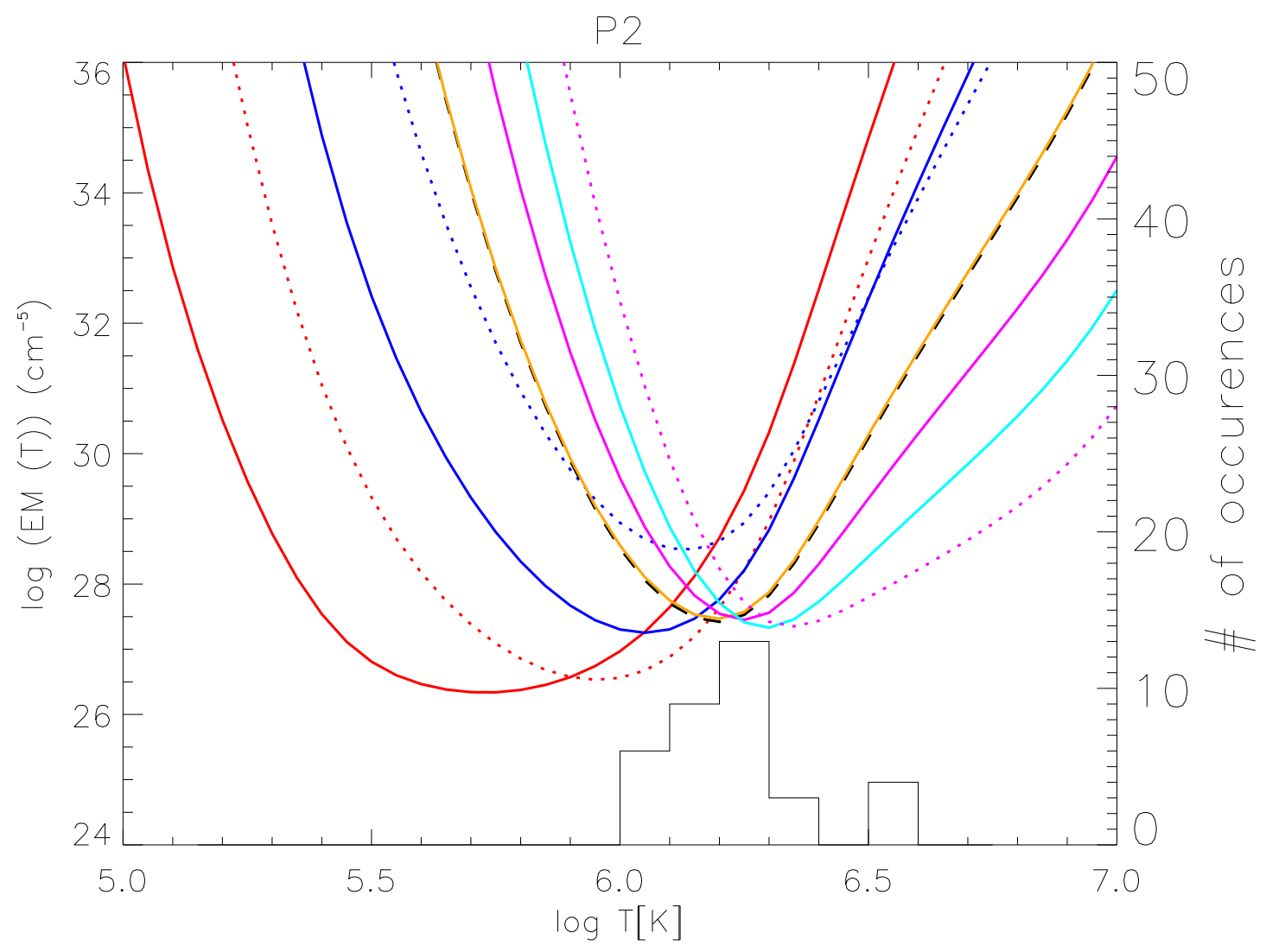

Fig. 12. Emission Measure (EM) loci curves for the bright point, corresponding to 'P2' (in Figs. 5 and 9), derived with spectral line observations during E2. The histograms representing number of crossings within a given temperature bin are plotted. No background/ foreground emission has been accounted for.
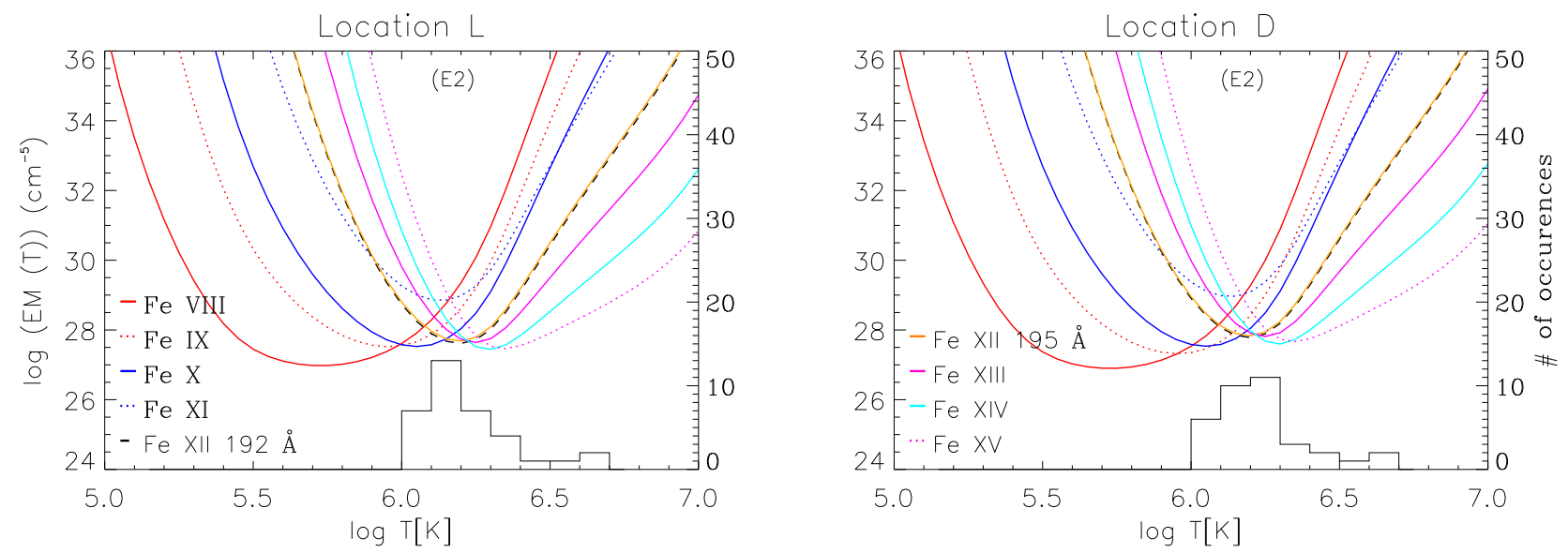

Fig. 13. Emission Measure (EM) loci curves for the TELs ('L', in left panel) and the hotter loops beneath ('D', in right panel) in E2, as identified in Fig. 9 Also plotted are the histograms representing number of crossings within a given temperature bin. No background/ foreground emission has been considered.

captured exactly by four exposures. This gave us the opportunity to understand the plasma parameters in this brightening point. Density estimates at P2 show that it has an intermediate value for Fe XII line. Further, the plasma at P2 has slightly higher temperature, $\log T[K]=6.20-6.30$. We emphasize that we have not incorporated background/foreground emission for temperature estimates for reasons explained in $\$ 3.4 .1 .1$
We have also compared the densities and temperatures obtained in the X-region with those obtained in TELs and other AR loops. The average electron densities in the TELs (location L of Fig. 9) exceed those in the X-region for all the four rasters. Similarly, the electron densities at location $\mathrm{D}$ is larger than those in the TELs. However, the densities at $\mathrm{L}$ is noted to be lower by an order of magnitude as reported by (see, e.g., Liu et al. 2011), using the Six line of CDS. We also find that just beneath the 

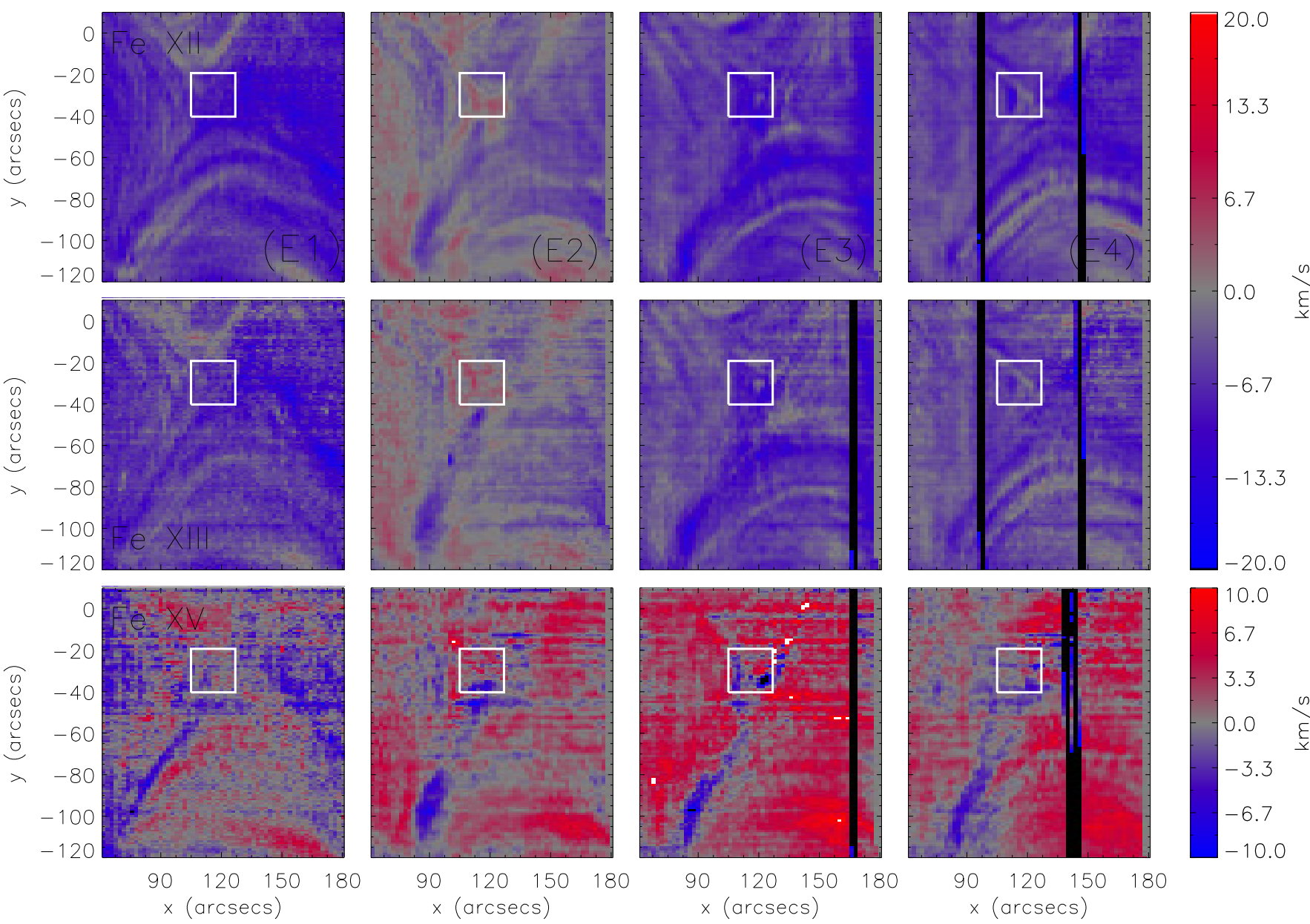

Fig. 14. Doppler maps corresponding to the X-region in Fe xII, Fe XIII and Fe xv lines. E2 is closer to the disk center.

X-region, a set of loops persist at $\sim 2 \mathrm{MK}$ and the TELs are at intermediate temperatures. Existence of such hot loops neighbouring the X-region has also been reported by Delannée \& Aulanier (1999); Glover et al. (2003); Pevtsov (2004); Balasubramaniam et al. (2005), though in the presence of eruptive events like flares or coronal mass ejections. The peak formation temperature in the loops belonging to the ARs individually is similar to that obtained at $\mathrm{P} 2$.

The Doppler velocity maps show a mixture of upflows and downflows for the comparatively cooler spectral lines in the Xregion. Near the disk center position, Fe XII, Fe XIII and Fe Xv show strong downflows but a mixture of zero velocities and blueshifts away from the center. These flows are $\sim 5-8 \mathrm{~km} \mathrm{~s}^{-1}$ upflows/downflows depending on the raster period. We note that the loops emanating from the reconnection show similar magnitude of Doppler velocities. With off-limb observations, Harra et al. (2003); Brosius (2006); Liu et al. (2011) have also reported similar bidirectional flows in such loops. The on-disk LOS velocities form the third mutually orthogonal component to the outflow speeds at the X-region noted above.

In the X-region, the average FWHM is $0.03 \AA$ which translates to about $27 \mathrm{~km} \mathrm{~s}^{-1}$ with the maximum being $\sim 62 \mathrm{~km} \mathrm{~s}^{-1}$ (corresponding to the brightest pixels) for Fe XII and Fe XIII. However, for Fe xv, the X-region show a FWHM of $\sim 0.05 \AA$ translating to $\sim 30 \mathrm{~km} \mathrm{~s}^{-1}$ whereas the maximum is $0.18 \AA$, equivalent to $\sim 115 \mathrm{~km} \mathrm{~s}^{-1}$. It is interesting to note that in case of Fexv, the enhanced FWHM region coincides with the dark $\mathrm{X}$-region in intensity map. We note that the non-thermal velocities are obtained considering the temperature of the X-region $(\log T[K]=6.20)$. The Doppler velocities and FWHM in P2 region is also comparable to those in the X-region. We highlight that the temperatures used for these estimates are representative of electron temperature of the plasma which can be considerably different than ion temperatures. This is particularly true in regions undergoing magnetic reconnection where equilibrium conditions are no longer valid. Considering that the ion temperatures are generally larger than the electron temperatures, it is apparent that the non-thermal velocities are somewhat overestimated here (Seely et al. 1997; Tu et al. 1998; Landi 2007).

The results obtained based on the xt-plots derived using the AIA observations combined with those from the EIS suggests that the TELs formed through the process of reconnection at the X-region formed between the two active regions. This study, for the first time, provides measurements of plasma parameters such as electron density, temperature, Doppler shifts as well as non-thermal velocities at X-region. We interpret that this is an example of homologous low-intensity magnetic reconnections occurring in TELs where the energy released is predominantly used in plasma flows and as kinetic energy source for the field lines snapping away and getting reoriented in some other direction. The intensity increment observed in AIA $193 \AA$ images is very small but not accompanied by any flares or coronal mass 

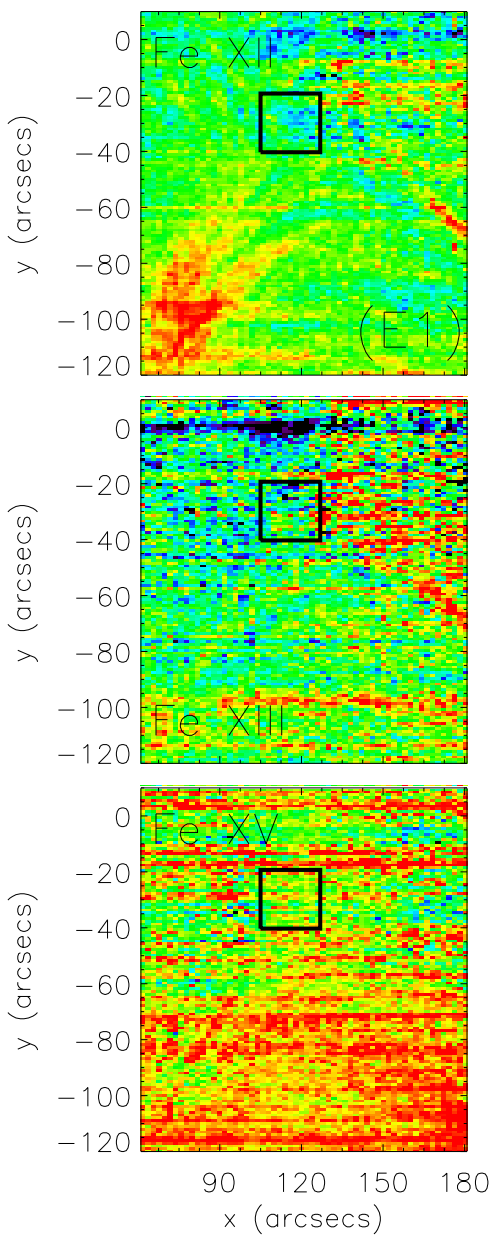
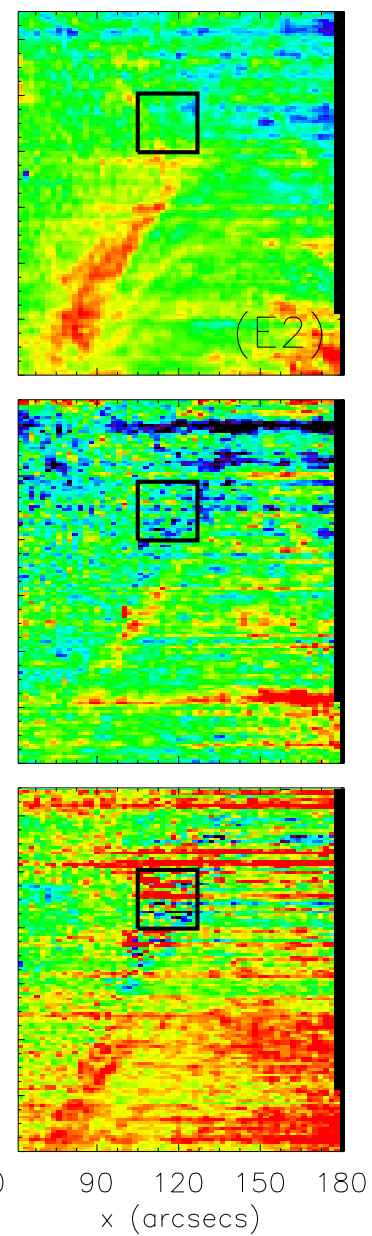
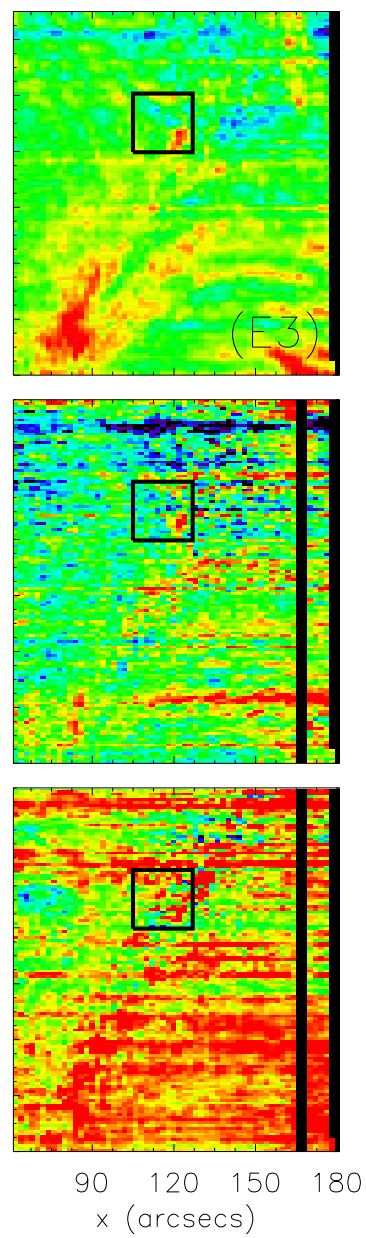
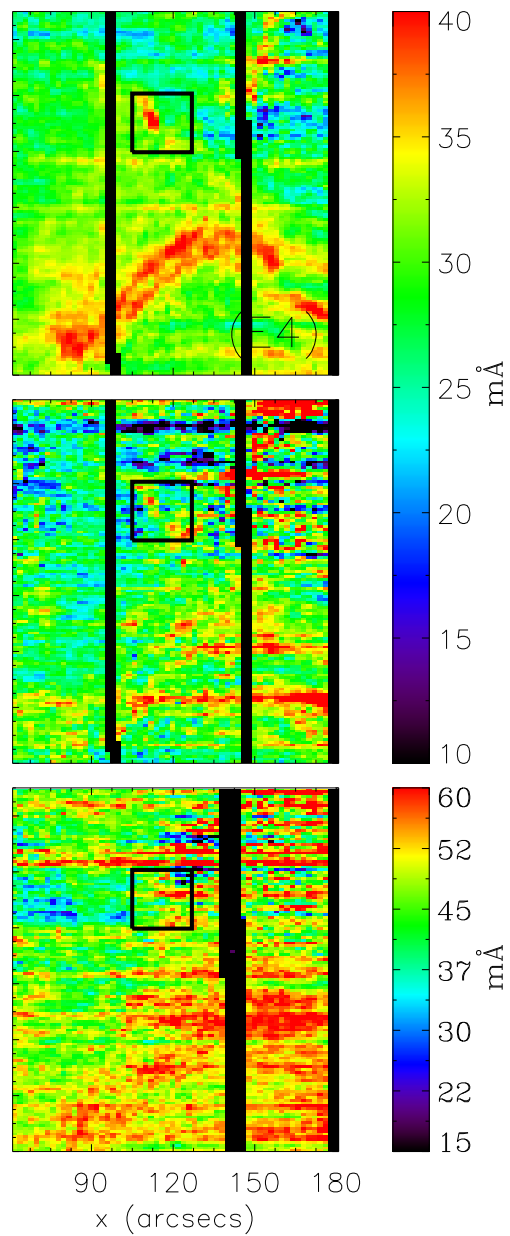

Fig. 15. FWHM maps in Fe XII, Fe xIII and Fe xv lines corresponding to the X-region.

ejections, unlike those reported by Khan \& Hudson (2000); Balasubramaniam et al. (2005). It is plausible that such small-scale reconnections of TELs steadily keep feeding energy into the solar corona, thereby being a source of heating. However, further studies are required to confirm how frequent such loop systems are in the solar atmosphere in a given time interval and estimation of energy released by them. The physical plasma parameters obtained in this study may provide constraints for MHD simulations of magnetic reconnections in future.

Acknowledgements. We thank the Referee for reading the manuscript carefully and provide valuable comments. We also thank Peter R. Young for various discussions and S. K. Solanki for his comments on an earlier version of the manuscript. This research is supported by the Max-Planck India Partner Group of MPS at IUCAA that is funded by MPG and DST. AIA and HMI data are courtesy of SDO (NASA). Facilities: SDO (AIA). Hinode is a Japanese mission developed and launched by ISAS/JAXA, collaborating with NAOJ as a domestic partner, NASA and STFC (UK) as international partners. Scientific operation of the Hinode mission is conducted by the Hinode science team organized at ISAS/JAXA. This team mainly consists of scientists from institutes in the partner countries. Support for the post-launch operation is provided by JAXA and NAOJ (Japan), STFC (UK), NASA, ESA, and NSC (Norway).

\section{References}

Babcock, H. W. 1961, ApJ, 133, 572

Balasubramaniam, K. S., Pevtsov, A. A., Neidig, D. F., et al. 2005, ApJ, 630, 1160

Boerner, P., Edwards, C., Lemen, J., et al. 2012, Sol. Phys., 275, 41
Brosius, J. W. 2006, ApJ, 636, L57

Brown, C. M., Feldman, U., Seely, J. F., Korendyke, C. M., \& Hara, H. 2008, ApJS, 176, 511

Chase, R. C., Krieger, A. S., Svestka, Z., \& Vaiana, G. S. 1976, in Space research XVI, ed. M. J. Rycroft, 917-922

Chen, J., Bao, S., \& Zhang, H. 2006, Sol. Phys., 235, 281

Chen, J., Bao, S. D., \& Zhang, H. Q. 2007, Advances in Space Research, 39, 1831

Crooker, N. U., Gosling, J. T., \& Kahler, S. W. 2002, Journal of Geophysical Research (Space Physics), 107, 1028

Culhane, J. L., Harra, L. K., James, A. M., et al. 2007, Sol. Phys., 243, 19

Del Zanna, G., Dere, K. P., Young, P. R., Landi, E., \& Mason, H. E. 2015, A\&A, 582, A56

Del Zanna, G. \& Mason, H. E. 2003, A\&A, 406, 1089

Del Zanna, G., O’Dwyer, B., \& Mason, H. E. 2011, A\&A, 535, A46

Delannée, C. \& Aulanier, G. 1999, Sol. Phys., 190, 107

Dere, K. P., Monsignori-Fossi, B. C., Landi, E., Mason, H. E., \& Young, P. R. 1996, in Bulletin of the American Astronomical Society, Vol. 28, American Astronomical Society Meeting Abstracts \#188, 961

Domingo, V., Fleck, B., \& Poland, A. I. 1995, Space Sci. Rev., 72, 81

Doschek, G. A., Warren, H. P., Mariska, J. T., et al. 2008, ApJ, 686, 1362

Fárník, F., Karlický, M., \& Švestka, Z. 1999, Sol. Phys., 187, 33

Fárník, F., Karlický, M., \& Švestka, Z. 2001, Sol. Phys., 202, 81

Fárník, F. \& Švestka, Z. 2002, Sol. Phys., 206, 143

Forbes, T. G. \& Acton, L. W. 1996, ApJ, 459, 330

Freeland, S. L. \& Handy, B. N. 1998, Sol. Phys., 182, 497

Glover, A., Harra, L. K., Matthews, S. A., \& Foley, C. A. 2003, A\&A, 400, 759

Golub, L., Deluca, E., Austin, G., et al. 2007, Sol. Phys., 243, 63

Harra, L. K., Matthews, S. A., \& van Driel-Gesztelyi, L. 2003, ApJ, 598, L59

Harrison, R. A., Sawyer, E. C., Carter, M. K., et al. 1995, Sol. Phys., 162, 233

Jordan, C. \& Wilson, R. 1971, in Astrophysics and Space Science Library,

Vol. 27, Physics of the Solar Corona, ed. C. J. Macris, 219

Khan, J. I. \& Hudson, H. S. 2000, Geophys. Res. Lett., 27, 1083 
Kosugi, T., Matsuzaki, K., Sakao, T., et al. 2007, Sol. Phys., 243, 3

Landi, E. 2007, The Astrophysical Journal, 663, 1363

Lemen, J. R., Title, A. M., Akin, D. J., et al. 2012, Sol. Phys., 275, 17

Liu, R., Wang, T.-J., Lee, J., et al. 2011, Research in Astronomy and Astrophysics, 11, 1209

O’Dwyer, B., Del Zanna, G., Mason, H. E., Weber, M. A., \& Tripathi, D. 2010, A\&A, 521, A21

Pesnell, W. D., Thompson, B. J., \& Chamberlin, P. C. 2012, Sol. Phys., 275, 3

Pevtsov, A. A. 2000, ApJ, 531, 553

Pevtsov, A. A. 2004, in IAU Symposium, Vol. 223, Multi-Wavelength Investigations of Solar Activity, ed. A. V. Stepanov, E. E. Benevolenskaya, \& A. G. Kosovichev, 521-524

Reale, F. 2014, Living Reviews in Solar Physics, 11

Schmelz, J. T., Kimble, J. A., \& Saba, J. L. R. 2012, ApJ, 757, 17

Schou, J., Borrero, J. M., Norton, A. A., et al. 2012a, Sol. Phys., 275, 327

Schou, J., Scherrer, P. H., Bush, R. I., et al. 2012b, Sol. Phys., 275, 229

Seely, J. F., Feldman, U., Schühle, U., et al. 1997, ApJ, 484, L87

Sheeley, Jr., N. R., Bohlin, J. D., Brueckner, G. E., et al. 1975, Sol. Phys., 40, 103

Shimojo, M., Narukage, N., Kano, R., et al. 2007, PASJ, 59, S745

Subramanian, S., Tripathi, D., Klimchuk, J. A., \& Mason, H. E. 2014, ApJ, 795, 76

Sun, J. Q., Cheng, X., Ding, M. D., et al. 2015, Nature Communications, 6, 7598

Svestka, Z., Krieger, A. S., Chase, R. C., \& Howard, R. 1977, Sol. Phys., 52, 69

Tripathi, D., Klimchuk, J. A., \& Mason, H. E. 2011, ApJ, 740, 111

Tripathi, D., Mason, H. E., Del Zanna, G., \& Young, P. R. 2010, A\&A, 518, A42

Tripathi, D., Mason, H. E., Dwivedi, B. N., del Zanna, G., \& Young, P. R. 2009, ApJ, 694, 1256

Tripathi, D., Solanki, S. K., Mason, H. E., \& Webb, D. F. 2007, A\&A, 472, 633

Tripathi, D., Solanki, S. K., Schwenn, R., et al. 2006, A\&A, 449, 369

Tsuneta, S. 1996, ApJ, 456, L63

Tsuneta, S., Acton, L., Bruner, M., et al. 1991, Sol. Phys., 136, 37

Tsuneta, S., Hara, H., Shimizu, T., et al. 1992, PASJ, 44, L63

Tu, C. Y., Marsch, E., Wilhelm, K., \& Curdt, W. 1998, ApJ, 503, 475

Vaiana, G. S., Krieger, A. S., Petrasso, R., Silk, J. K., \& Timothy, A. F. 1974, in Proc. SPIE, Vol. 44, Instrumentation in Astronomy II, ed. D. H. Menzel, L. Larmore, \& D. Crawford, 185-205

Viall, N. M. \& Klimchuk, J. A. 2011, ApJ, 738, 24

Wang, J., Zhang, Y., Zhou, G., et al. 2007, Sol. Phys., 244, 75

Warren, H. P., Ugarte-Urra, I., Young, P. R., \& Stenborg, G. 2011, ApJ, 727, 58

Yokoyama, M. \& Masuda, S. 2009, Sol. Phys., 254, 285

Yokoyama, M. \& Masuda, S. 2010, Sol. Phys., 263, 135

Yokoyama, T., Akita, K., Morimoto, T., Inoue, K., \& Newmark, J. 2001, ApJ, 546, L69

Young, P. R., Del Zanna, G., Mason, H. E., et al. 2007, PASJ, 59, 857

Young, P. R., O'Dwyer, B., \& Mason, H. E. 2012, ApJ, 744, 14

Young, P. R., Watanabe, T., Hara, H., \& Mariska, J. T. 2009, A\&A, 495, 587 


\begin{tabular}{|c|c|c|c|c|c|c|c|}
\hline & 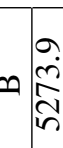 & & & & $\frac{t}{\vec{\theta}}$ & $\vec{\infty}$ & 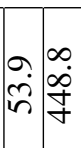 \\
\hline & $\tilde{\Sigma}) 1$ & 1 & \begin{tabular}{l|l}
1 & 1
\end{tabular} & 1 & ' & ' & $\begin{array}{ll}1 & 1\end{array}$ \\
\hline & & & 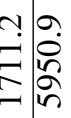 & $\stackrel{\sim}{\check{n}}$ & $\begin{array}{l}n \\
0 \\
0 \\
\varnothing \\
0 \\
0\end{array}$ & $\begin{array}{l}n \\
\vdots \\
0 \\
1 \\
m\end{array}$ & 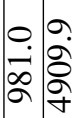 \\
\hline & & & 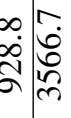 & $\frac{n}{\stackrel{n}{a}}$ & 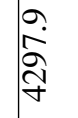 & $\begin{array}{l}\hat{a} \\
\grave{\lambda} \\
\vec{\lambda}\end{array}$ & 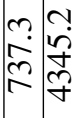 \\
\hline & & & 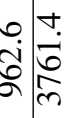 & $\begin{array}{l}\overrightarrow{0} \\
\exists\end{array}$ & $\begin{array}{l}n \\
\tilde{y} \\
\tilde{m}\end{array}$ & 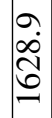 & 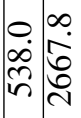 \\
\hline & & & 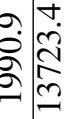 & تَ & $\begin{array}{l}\hat{a} \\
\dot{n} \\
\ddot{n}\end{array}$ & $\begin{array}{l}0 \\
\dot{r} \\
\bar{N}\end{array}$ & 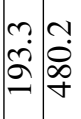 \\
\hline & $\tilde{\Sigma})$. & I & ' & ' & ' & ' & 1.1 \\
\hline & & & & 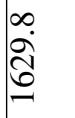 & 茂 & $\begin{array}{l}\infty \\
\dot{\rho} \\
\tilde{m} \\
m\end{array}$ & 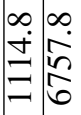 \\
\hline & & & 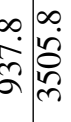 & $\begin{array}{l}\hat{\sigma} \\
\grave{0} \\
\approx\end{array}$ & $\begin{array}{l}m \\
? \\
\tilde{f} \\
m\end{array}$ & 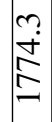 & 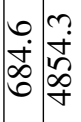 \\
\hline & & & & 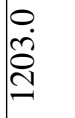 & $\begin{array}{l}0 \\
\dot{b} \\
\vec{f}\end{array}$ & $\begin{array}{l}0 \\
\dot{0} \\
\vdots \\
0 \\
0\end{array}$ & 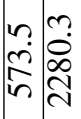 \\
\hline & & & 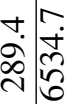 & 家 & $\begin{array}{l}\text { ĩ } \\
\text { Sิ }\end{array}$ & 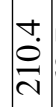 & $\dot{s}: \frac{m}{8}$ \\
\hline & - & & \begin{tabular}{l|l}
$\underset{n}{*}$ \\
$\stackrel{4}{n}$
\end{tabular} & $\hat{\imath}$ & $\frac{m}{\stackrel{\Xi}{\sim}}$ & $\begin{array}{l}n \\
\infty \\
0 \\
0 \\
0\end{array}$ & 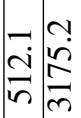 \\
\hline & & & 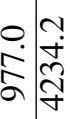 & 苞 & 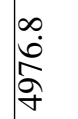 & & 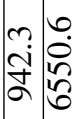 \\
\hline & & & 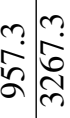 & $\begin{array}{l}\infty \\
\text { in } \\
\tilde{n}\end{array}$ & 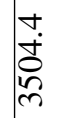 & $\begin{array}{l}\hat{\jmath} \\
\grave{i} \\
\stackrel{2}{2}\end{array}$ & 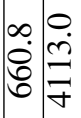 \\
\hline & & & 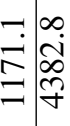 & $\begin{array}{l}\infty \\
i \\
\\
\beth\end{array}$ & 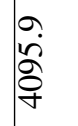 & 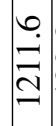 & 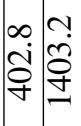 \\
\hline & $\sim \mid \begin{array}{l}0 \\
0 \\
0 \\
0 \\
0\end{array}$ & & 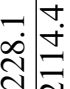 & 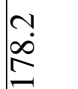 & $\begin{array}{l}\infty \\
\infty \\
\infty \\
\delta\end{array}$ & $\begin{array}{l}\infty \\
0 \\
0 \\
0\end{array}$ & 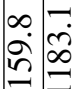 \\
\hline & $\stackrel{\Sigma}{\Sigma}$, & I & ' & I & ' & 1 & 1.1 \\
\hline$\vec{I}$ & & $\stackrel{m}{\stackrel{\sim}{N}}$ & 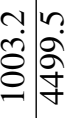 & $\begin{array}{l}\hat{a} \\
\hat{a} \\
\underline{2}\end{array}$ & 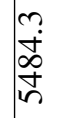 & $\begin{array}{l}m \\
\stackrel{m}{a} \\
m\end{array}$ & 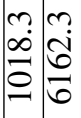 \\
\hline & & 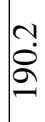 & 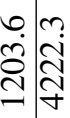 & 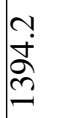 & 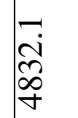 & & 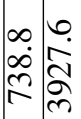 \\
\hline & $\alpha \mid \begin{array}{l}0 \\
\dot{0} \\
0 \\
n\end{array}$ & 8 & b. & 年 & 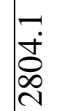 & & 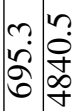 \\
\hline $\mid$\begin{tabular}{|l}
$\mathscr{\Xi}$ \\
$\Xi$ \\
0 \\
$\underline{L}$ \\
\end{tabular} & & & & 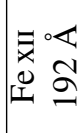 & $\mid \begin{array}{ll}x^{\circ} & < \\
0 & 0 \\
1 & 0\end{array}$ & & 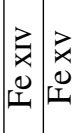 \\
\hline
\end{tabular}




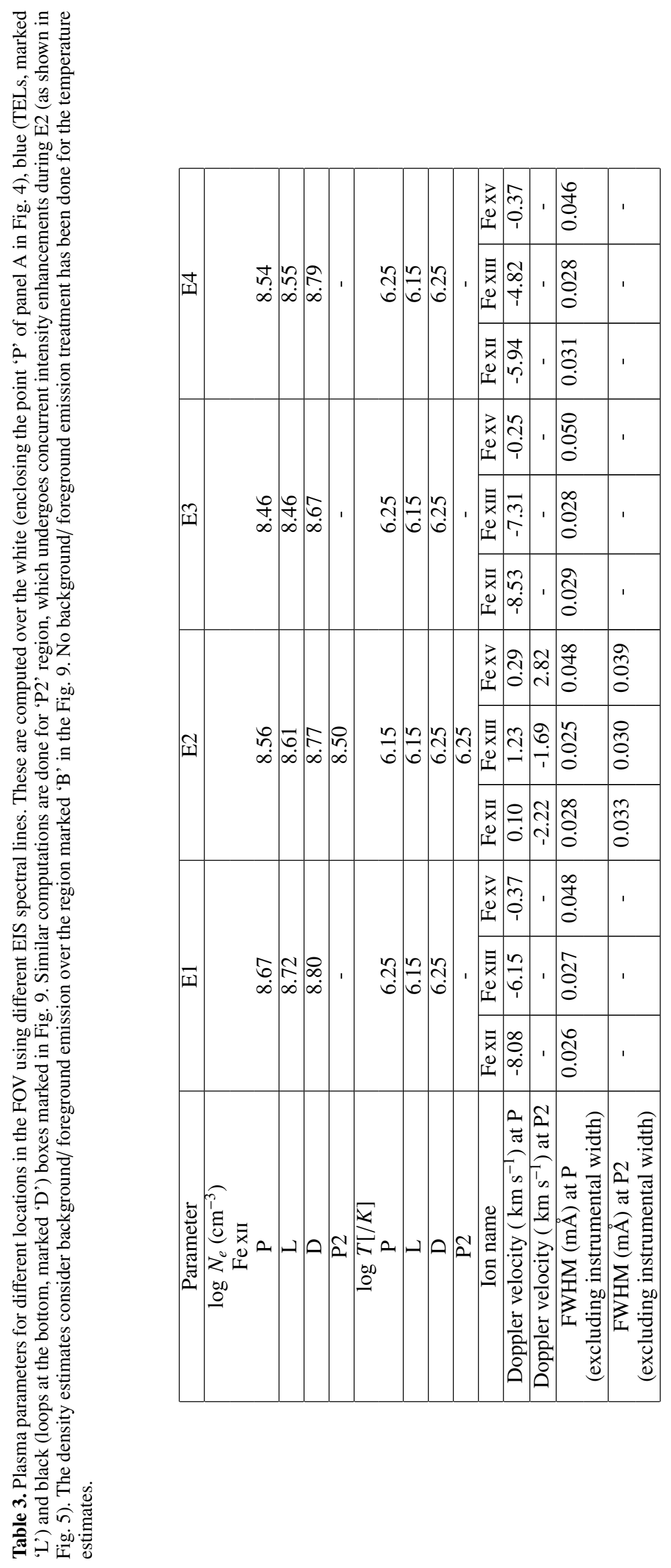

